\title{
Antiangiogenic immunotherapy suppresses desmoplastic and chemoresistant intestinal tumors in mice
}

\author{
Simone Ragusa, ${ }^{1,2}$ Borja Prat-Luri, ${ }^{1,2}$ Alejandra González-Loyola, ${ }^{1,2}$ Sina Nassiri, ${ }^{3,4}$ Mario Leonardo Squadrito, ${ }^{4}$ Alan Guichard, ${ }^{4}$ \\ Sabrina Cavin, ${ }^{5}$ Nikolce Gjorevski, ${ }^{6}$ David Barras, ${ }^{3}$ Giancarlo Marra, ${ }^{7}$ Matthias P. Lutolf, ${ }^{6}$ Jean Perentes, ${ }^{5}$ Emily Corse, ${ }^{8}$ \\ Roberta Bianchi, ${ }^{8}$ Laureline Wetterwald, ${ }^{1,2}$ Jaeryung Kim, ${ }^{1,2}$ Guillermo Oliver, ${ }^{9}$ Mauro Delorenzi, ${ }^{1,2,3}$ Michele De Palma, ${ }^{4}$ \\ and Tatiana V. Petrova ${ }^{1,2}$ \\ 'Department of Oncology, University of Lausanne and CHUV, Epalinges, Switzerland. 'Ludwig Institute for Cancer Research Lausanne, Epalinges, Switzerland. "3ioinformatics Core Facility, Swiss Institute \\ of Bioinformatics, Lausanne, Switzerland. ${ }^{4}$ Swiss Institute for Experimental Cancer Research, Ecole Polytechnique Federale de Lausanne (EPFL), Lausanne, Switzerland. ${ }^{5}$ Division of Thoracic Surgery, \\ CHUV, Lausanne, Switzerland. 'Institute of Bioengineering, School of Life Sciences, EPFL, Lausanne, Switzerland. 'Institute of Molecular Cancer Research, University of Zurich, Zurich, Switzerland. ${ }^{8}$ Roche \\ Innovation Center Zurich, Roche Pharmaceutical Research and Early Development, (pRED), Schlieren, Switzerland. 'ंCenter for Vascular and Developmental Biology, Feinberg Cardiovascular and Renal \\ Research Institute, Feinberg School of Medicine, Northwestern University, Chicago, Illinois, USA.
}

\begin{abstract}
Mutations in APC promote colorectal cancer (CRC) progression through uncontrolled WNT signaling. Patients with desmoplastic CRC have a significantly worse prognosis and do not benefit from chemotherapy, but the mechanisms underlying the differential responses of $A P C$-mutant CRCs to chemotherapy are not well understood. We report that expression of the transcription factor prospero homeobox 1 (PROX1) was reduced in desmoplastic APC-mutant human CRCs. In genetic Apc-mutant mouse models, loss of Prox1 promoted the growth of desmoplastic, angiogenic, and immunologically silent tumors through derepression of Mmp14. Although chemotherapy inhibited Prox1-proficient tumors, it promoted further stromal activation, angiogenesis, and invasion in Prox1-deficient tumors. Blockade of vascular endothelial growth factor A (VECFA) and angiopoietin-2 (ANGPT2) combined with CD40 agonistic antibodies promoted antiangiogenic and immunostimulatory reprogramming of Prox1-deficient tumors, destroyed tumor fibrosis, and unleashed T cell-mediated killing of cancer cells. These results pinpoint the mechanistic basis of chemotherapy-induced hyperprogression and illustrate a therapeutic strategy for chemoresistant and desmoplastic CRCs.
\end{abstract}

\section{Introduction}

Colorectal cancer (CRC) is one of the leading causes of cancerrelated mortality worldwide (1). Patients with stage III or IV CRC have a high risk of recurrence and are typically treated with surgery and 5-FU-based adjuvant chemotherapy alone (stage III), or combined with targeted therapy such as anti-VEGF ( $\alpha$-VEGF) or $\alpha$-EGFR antibodies (stage IV). Chemotherapy increases the overall survival by up to $15 \%$, yet many patients do not respond or relapse, highlighting the need for the development of new therapeutic strategies. Likewise, targeted blockade of the oncogenic EGFR pathway or VEGF-driven angiogenesis have shown effectiveness in advanced CRC, but most clinical responses are transitory. Most recently, programmed cell death 1 (PD-1) immune checkpoint blockade was approved for the treatment of CRCs with defective mismatch repair. However, the treatment is not

\section{Related Commentary: p. 1093}

Authorship note: BPL and AGL contributed equally to this work. Conflict of interest: RB is a current Roche employee and EC is a former Roche employee. TVP and MDP received research funding from Roche. Copyright: () 2020, American Society for Clinical Investigation. Submitted: April 15, 2019; Accepted: November 20, 2019; Published: February 4, 2020. Reference information: / Clin Invest. 2020;130(3):1199-1216. https://doi.org/10.1172/JCl129558. effective in patients with microsatellite-stable (MSS) CRC, which accounts for the majority of cases $(2,3)$. A better understanding of the mechanisms underlying primary and acquired resistance to current therapies in CRC and the development of novel combinatorial approaches are urgently needed to improve the treatment of this common and lethal disease.

One of the key factors accounting for highly variable outcomes is the molecular heterogeneity of CRC. Recently, 2 molecular classifications were established: consensus molecular subtypes 1-4 (CMS14), based on total tumor transcriptomes, and CRC-intrinsic subtypes (CRIS, CRIS-A-E), based on cancer cell transcriptomes (4-6). CMS1 (14\% of CRC cases) is characterized by microsatellite instability (MSI), high levels of mutations, strong immune cell activation, low WNT signaling, and a good prognosis; CMS2 (37\%) is epithelium rich, with marked canonical WNT pathway activation and proliferative signaling; CMS3 (13\%) displays metabolic dysregulation and intermediate WNT signaling; CMS4 (23\%) is characterized by low WNT signaling, a high proportion of stromal cells, prominent angiogenesis, and a poor prognosis (4). Causative molecular alterations are more obvious in CMS1 and CMS3 groups, which show hypermutability (94\% and 28\%) and a high rate of BRAF (CMS1) or KRAS (CMS3) mutations. However, the spectrum of driver mutations (APC, KRAS, TP53) is similar in MSS CMS2 and CMS4 subtypes, and it remains unclear why patients with CMS4 tumors have faster metastatic progression and shorter overall survival (4). 
Comparison of CMS and CRIS classifications revealed that most CMS1 and CMS3 tumors belong to the CRIS-A subtype, enriched for MSI or KRAS mutations, whereas the CMS2 subtype comprises 3 CRIS groups with high WNT signaling but also distinct features: CRIS-C has high EGFR pathway activity; CRIS-D is enriched for IGF2 overexpression, and CRIS-E has Paneth cell-like characteristics. A CRIS-B subtype was also identified, with TGF- $\beta$ pathway activation, epithelial-to-mesenchymal transition (EMT), and poor prognosis, whereas the CMS4 subtype was distributed among all CRIS subtypes, confirming previous observations that the CMS4 transcriptome is dominated by stromal contribution (5-9). Analysis of clinical responses to adjuvant chemotherapy in patients with stage III CRC revealed a benefit for epithelium-rich CMS2 and CMS3 subgroups, but not for the CMS1 or CMS4 subgroups $(7,10,11)$. However, the molecular mechanism underlying this differential response pattern is not fully understood.

In addition to molecular subtypes, a high degree of $\mathrm{CD}^{+}$and $\mathrm{CD}^{+} \mathrm{T}$ cell infiltration into the primary tumor is associated with a better prognosis in both MSI and microsatellite stable (MSS) CRCs, where it strongly predicts the time to recurrence, overall survival, and disease-free survival (12). High T cell infiltration is associated with MSI status in CRCs, and it is also observed in $20 \%$ of MSS CRC tumors. The molecular causes that mediate $\mathrm{T}$ cell exclusion in the majority of MSS CRCs are not completely understood. Such exclusion is at least in part a result of the significantly lower mutational burden of MSS CRCs (13), however, additional active mechanisms preventing intratumoral cytotoxic $\mathrm{T}$ lymphocyte (CTL) accumulation and activation probably exist, and antitumor T cell immunity can be elicited in preclinical models of MSS CRC (14). Given that current checkpoint inhibitors are ineffective in most patients with MSS CRC, it is essential to understand the interactions between cancer cells and the tumor microenvironment (TME) of different CRC subtypes in order to develop new targeted interventions capable of shifting the balance toward a tumor-rejecting response.

Targeting the tumor blood vasculature has emerged as a promising approach to achieve this goal. Continuous production of the angiogenic and vessel-destabilizing factors VEGFA and angiopoietin-2 (ANGPT2) impairs the alignment of tumor endothelial cells (ECs) and pericytes, increases vessel permeability, and reduces tumor perfusion. Such impaired tumor blood vessels promote an immunosuppressive environment by hindering the efficient extravasation and functioning of CTLs (15). The reversal of tumor vessel abnormality or "vascular normalization" has been shown to increase the penetration of activated immune cells (16). In a murine breast cancer model, the simultaneous blockade of VEGFA and ANGPT2 using the bispecific blocking antibody A2V was highly effective in normalizing the tumor vasculature, reprograming the TME, and increasing the efficacy of immune checkpoint blockade (17). Also, angiogenic blockade promoted the formation of high endothelial venule-like (HEV-like) vessels and improved the response to immune therapies in a genetic model of pancreatic neuroendocrine tumor $(18,19)$.

Cancer-associated fibroblasts (CAFs) are another essential TME component that both foster tumor angiogenesis and directly limit a productive antitumor response (15). Therapeutic blockade of the profibrotic and immunosuppressive factor TGF- $\beta$ has recently been shown to increase sensitivity to $\mathrm{PD}-1 /$ programmed cell death ligand 1 (PD-1/PD-L1) immune checkpoint blockade in genetic models of CRC (14). In desmoplastic pancreatic ductal adenocarcinoma, tumor anticancer immunity can be efficiently unleashed through the activation of CD40 signaling $(20,21)$.

Here, we investigated the responses to chemotherapy and immunotherapy of prospero homeobox 1 (PROX1)- proficient $\left(\mathrm{PROX}^{\mathrm{hi}}\right)$ and PROX1-deficient (PROX $1^{\mathrm{lo}}$ ) models of MSS Apcmutant intestinal tumors. PROX1 is a transcriptional repressor that is overexpressed in a subset of MSS CRCs with high WNT signaling (22). Under physiological conditions, PROX1 specifies cell fate and maintains the expression of lineage-specific genes in lymphatic ECs, cardiomyocytes, hepatocytes, and certain neuronal populations (23-27). PROX1 is not expressed in normal intestinal stem cells and is ectopically induced in intestinal epithelium upon oncogenic transformation in response to supraphysiological and prolonged WNT activation (22). It promotes metabolic adaptation of transformed intestinal stem cells and enhances tumor growth in preclinical CRC models $(28,29)$. Here, we report that PROX1 restrained stromal activation in $\mathrm{WNT}^{\text {hi }}$ tumors and licensed the chemosensitive phenotype. Surprisingly, we found that chemotherapy potentiates desmoplasia, angiogenesis, and aggressive growth of Prox1-deficient tumors and identified the metalloprotease MMP14 as a target of PROX1 that promotes such a response. We further show that the combined blockade of angiogenesis and CD40-driven immune activation was an efficient alternative treatment approach that unleashed $\mathrm{T}$ cell-mediated killing of cancer cells in chemoresistant Apc-mutant tumors. We believe our results have broad implications for the development of novel therapies to treat $A P C$-mutant MSS CRCs, which represent the majority of all CRC cases.

\section{Results}

High PROX1 expression is associated with a better prognosis and low stromal content in human CRCs. PROX1 is highly expressed in a subpopulation of CRC stem/progenitor cells (CSCs) in human WNT $^{\text {hi }}$ MSS tumors, but not in WNT ${ }^{\text {lo }}$ MSI tumors (28). We and others found that PROX1 sustains the metabolic fitness of CSCs and potentiates the growth of colon cancer cells in 3D organoid and mouse models $(28,29)$. To further study the clinical relevance of these observations, we analyzed the association of PROX1 with clinical outcomes in 444 patients from a prognostically annotated CRC gene expression data set (Gene Expression Omnibus [GEO] GSE39582) (30). Surprisingly, we found that high levels of PROX1 were associated with improved overall survival (Figure 1A). Analysis of pathways associated with PROX1 expression further showed that high PROX1 expression was positively correlated with WNT activation (Figure 1B), in agreement with previous data (28). Accordingly, PROX1 expression was highest in the WNT ${ }^{\text {hi }}$ CMS2 group (Figure 1C). Our clinical outcome analysis differed from the results of a previous histopathological study (31), therefore, we also analyzed tumors classified according to 5 intrinsic subtypes (6) and found that PROX1 expression was significantly higher in the WNT ${ }^{\text {hi }}$ CRIS-C, -D, and -E subtypes compared with expression in the poor-prognosis CRIS-B subtype (Figure 1D).

PROX1 expression was negatively correlated with a stromal gene signature (Figure 1E), consistent with decreased PROX1 
A

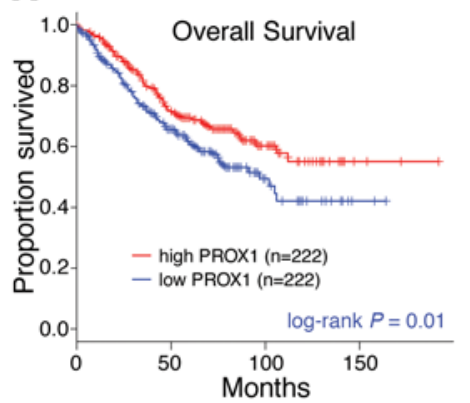

D

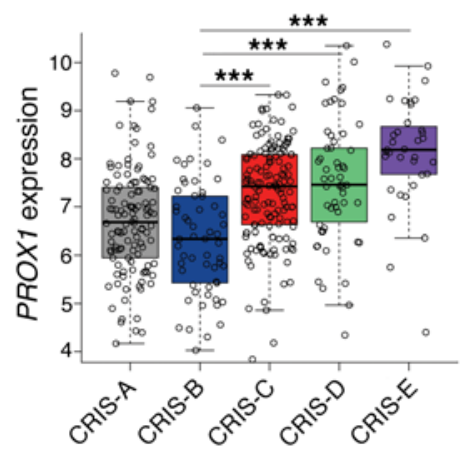

B

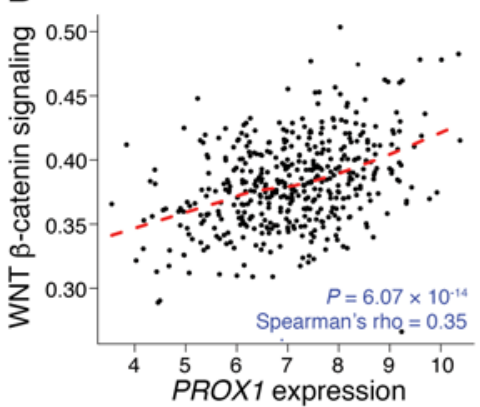

E

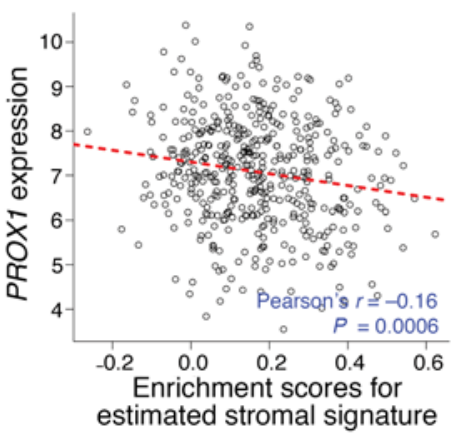

C

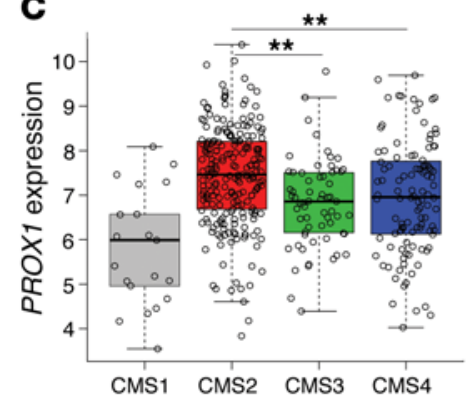

$\mathbf{F}$

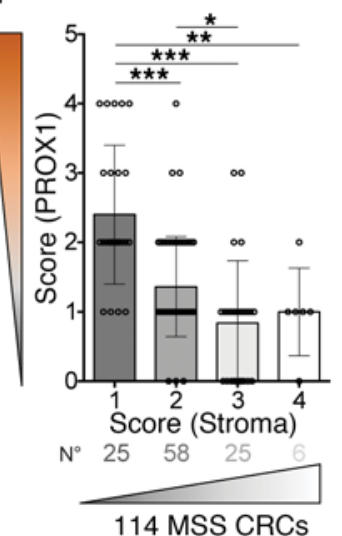

G

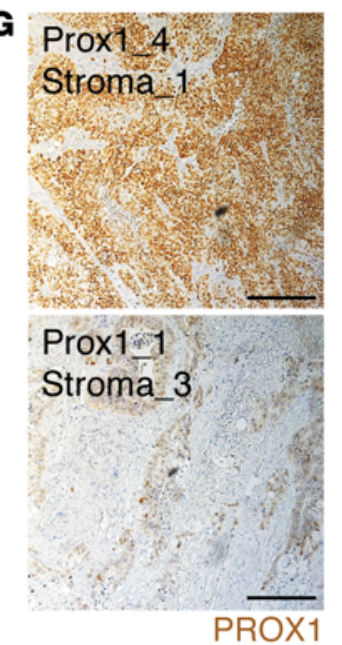

Figure 1. High PROX1 expression is associated with better clinical outcomes and low stromal content. (A) Kaplan-Meier overall survival curves for patients with high or low PROX1 levels in MSS CRC tumors (CEO GSE39582; $n=444$ ). Progression-free survival showed the same trend but did not reach significance $(P=0.3)$. (B) PROX1 expression correlation with WNT pathway activation in MSS CRCs (GSE39582; $n=444)$. Dashed line indicates the locally estimated scatterplot smoothing (LOESS) fit. (C) PROX1 expression in CRC CMS subgroups (CSE39582; $n=409)$. CMS1 MSI-like ( $n=21$ ); CMS2 high WNT signaling $(n=217)$; CMS3 KRAS-mutant and metabolic alterations $(n=63)$; CMS4 TCF- $\beta$-driven stromal and angiogenic activation $(n=108)$. $P<0.001$, by 1 -way ANOVA with Tukey's multiple comparisons test. $P<0.001$, for CMS2 verus CMS1; $P=0.001$, for CMS2 versus CMS3; $P=0.003$, for CMS2 versus CMS4. (D) PROX1 expression in intrinsic CRIS subtypes. CRIS-A: BRAF- or KRAS-mutated, secretory ( $n=88$ ); CRIS-B TCF- $\beta$ signaling, EMT features ( $n=59$ ); CRIS-C KRAS WT, high ERBB/EGFR pathway activity, MYC copy number gains ( $n=119)$; CRIS-D: high WNT, IGF2 amplification, and FGFR autocrine stimulation ( $n=96)$; CRIS-E: high WNT, Paneth-like phenotype, and TP53-mutations $(n=82)$ in GSE39582 $(n=444)$. $P<0.001$, by 1-way ANOVA with Tukey's multiple comparisons test. (E) PROX1 expression negatively correlated with a tumor stromal signature in MSS CRCs (CSE39582; $n=444$ ). The stromal gene signature is from ref. 80. Enrichment was computed using single-sample GSEA (81). Dashed line indicates the linear regression fit. (F) Scatterplot of the negative correlation between scores for PROX1 protein nuclear expression levels in tumor cells and for stromal content over the total tumor area, in MSS primary CRC samples $(n=114)$. (C) Representative images of CRC adenocarcinomas with high and low PROX1 scores. PROX1 staining (brown) and DNA counterstaining (blue) are shown. Scale bars: $200 \mu \mathrm{m}$.

expression in desmoplastic CMS4 CRCs (Figure 1C). For an independent validation of these data, we quantified PROX1 protein and stromal content of 114 primary MSS CRCs. We found that tumors with low nuclear PROX1 levels had greater stromal content (Figure 1, F and G, and Supplemental Table 1; supplemental material available online with this article; https://doi.org/10.1172/ JCI129558DS1). These data indicate that low PROX1 expression is associated with reduced WNT activation, high stromal content, and worse clinical outcomes in human MSS CRCs.

Inactivation of Prox1 attenuates WNT signaling in mouse intes-

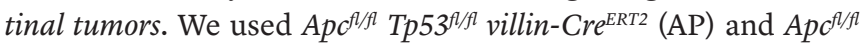
$T p 53^{f / / l}$ Prox $1^{f / / f}$ villin-Cre $e^{E R T 2}$ (APP) mice, in which Apc, Tp53, and Prox1 can be conditionally inactivated in the intestinal epithelium to induce tumors, to determine whether Prox 1 expression in cancer cells regulates tumor stromal content and properties. We induced tumors in both AP and APP mice by microinjection of tamoxifen into the cecum. With this approach, ectopic hyperactivation of WNT signaling occurs in a limited number of intestinal epithelial cells and leads to the development of a single, large, and frequently invasive cecal tumor that does not interfere with intestinal transit, thus allowing for the study of advanced tumors (Figure 2A and Supplemental Figure 1A).

Abnormally high WNT signaling is a main driver of cancer cell proliferation, tumor growth, and dedifferentiation in Apc-mutant CRC models $(32,33)$. We found that APP mouse tumors were significantly smaller than AP mouse tumors 4 weeks after tamoxifen microinjection (Figure 2B), in agreement with previous observations in Apc ${ }^{\mathrm{min} /+}$ Prox $1^{f / / l}$ villin-Cre adenomas (22). Expression of the WNT target genes leucine-rich repeat-containing G proteincoupled receptor 5 (Lgr5), lymphoid enhancer binding factor 1 (Lef1), ring finger protein 43 (Rnf43), and Eph receptor B2 (EphB2) (34-36) was decreased but not completely abolished upon loss of 
A

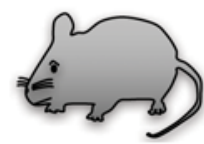

APIAPP

$\prod \begin{aligned} & \text { Intracecal } \\ & \text { tamoxifen }\end{aligned}$

Localized

deletion

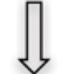

Single

tumor

$\mathrm{AP}$ - $A p c^{f l / f l} ; p 53^{f / f t}$

APP- $A p c^{f / f f l} ; p 53^{f / f f l} ; \operatorname{Prox} 1^{f / f t}$
B
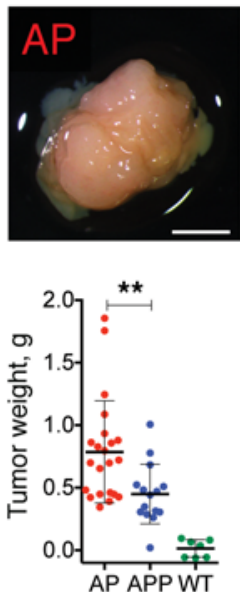
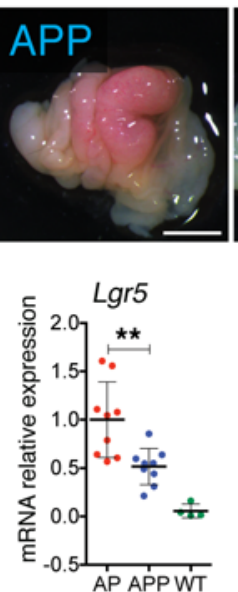
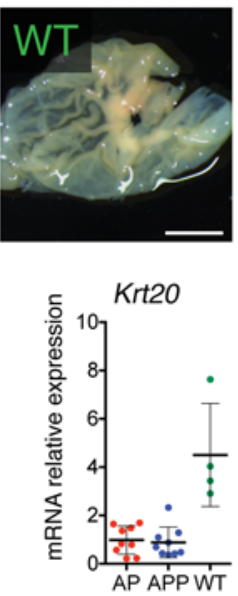

C

EPITHELIAL_MESENCHYMAL_TRANSITION ANGIOGENESIS UV_RESPONSE_DN MYOGENESIS OXIDATIVE_PHOSPHORYLATION FATTY_ACID_METABOLISM ESTROGEN_RESPONSE_LATE TNFA_SIGNALING_VIA_NFKB ALLOGRAFT REJECTION IL6 JAK STAT3 SIGNALING INTERFERON_GAMMA_RESPONSE INTERFERON_ALPHA_RESPONSE

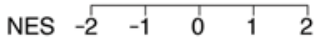

AP APP
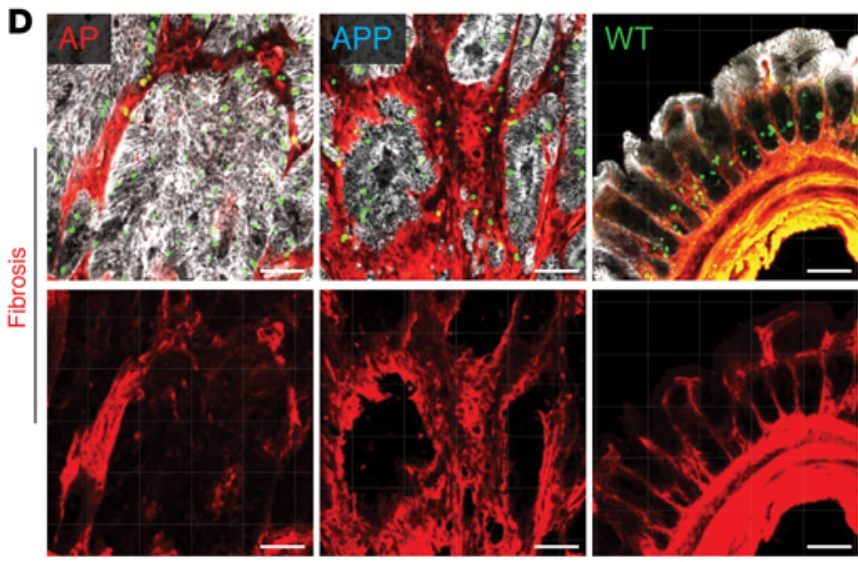

PH3 SMA E-cadherin

$\mathbf{E}$
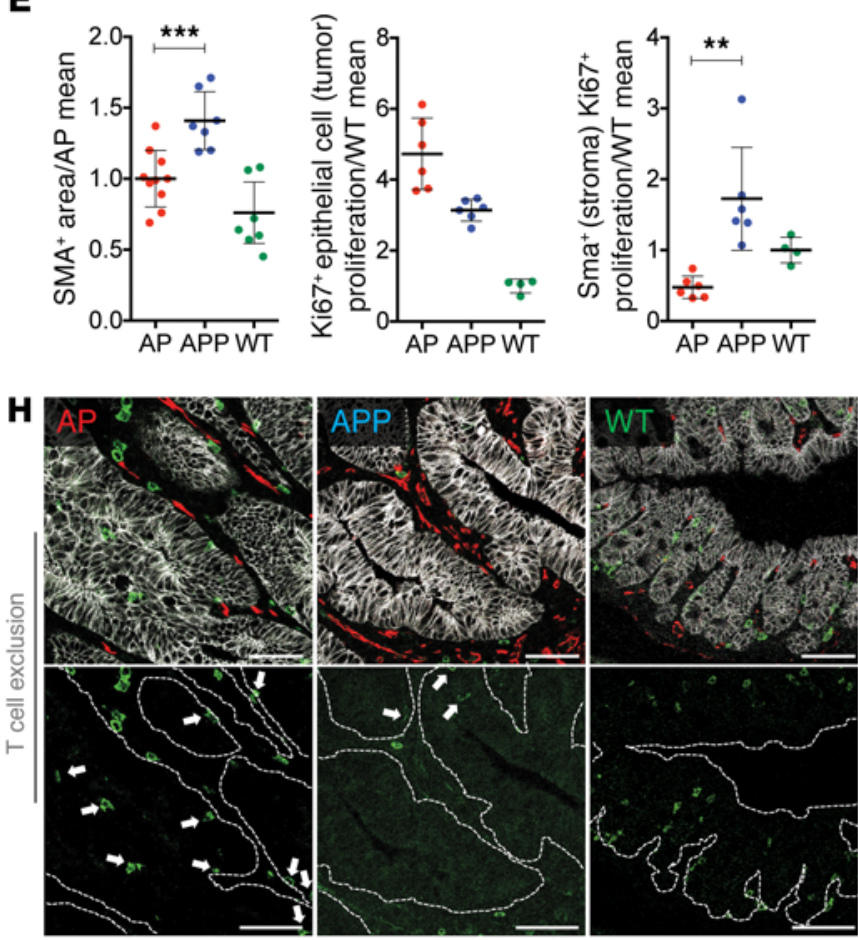

CD8a VE-cadherin E-cadherin
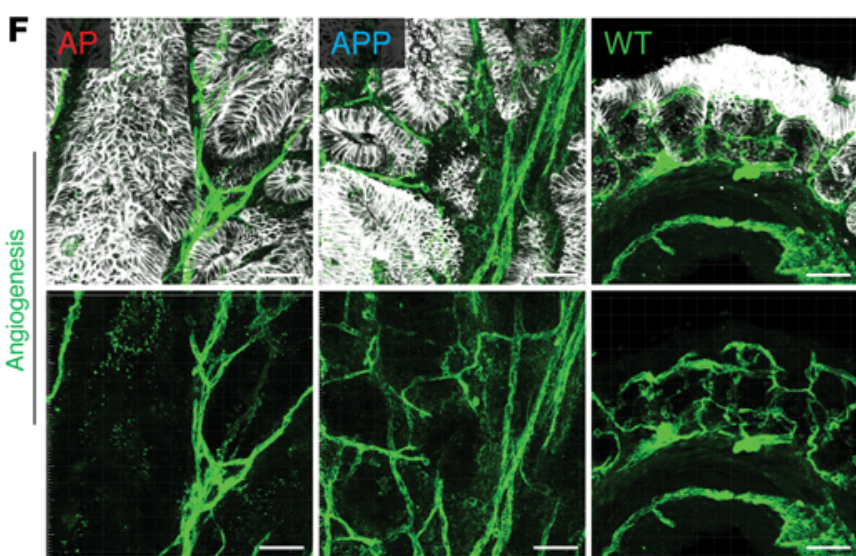

CD31 E-cadherin
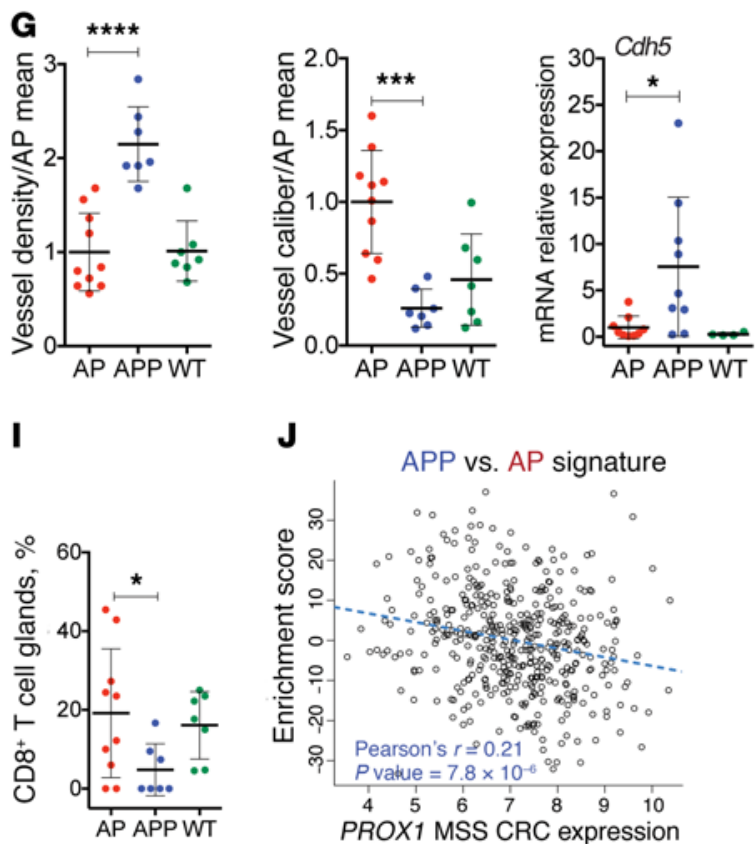

J

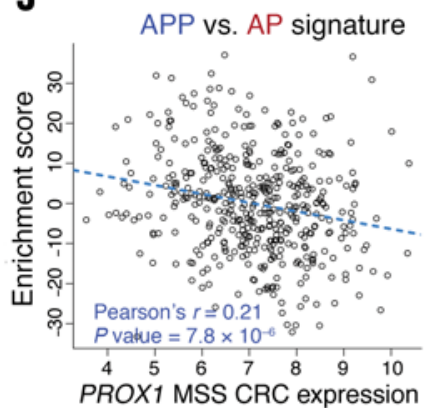


Figure 2. Inactivation of Prox1 generates desmoplastic, angiogenic, and T cell-excluded tumors. (A) Apc $c^{f / f l} T p 53^{f / f f}$ villin-Cre $e^{E R T 2}$ (AP) and $A p c^{f / f l} T p 53^{f / f f l}$ Prox $1^{\text {fIfl }}$ villin-Cre ${ }^{E R T 2}$ (APP) tumor models used in the study. (B) Appearance and weights of tumors and WT cecum and quantitative reverse transcription PCR (qRT-PCR) data for Lgr5 and Krt20. AP ( $n=22)$; APP ( $n=15)$; WT $(n=7)$. QRT-PCR data were normalized to the AP mean. AP or APP $(n=$ 9); WT $(n=4)$. Scale bars: $4 \mathrm{~mm}$. (C) Pathways enriched in APP versus AP transcriptomes ( $n=6$ per genotype). NES, normalized enrichment score. (D) APP tumors were desmoplastic. 3D reconstructions of tumor and WT cecum thick slices. Images show staining for PH3 (green), $\alpha$-SMA (red), and E-cadherin (white). Scale bars: $50 \mu \mathrm{m}$. (E) Quantification of proliferation of stromal and tumor epithelial cells. The $\alpha-\mathrm{SMA}^{+}$area from $\mathbf{D}$ was normalized to the total tumor area and the AP mean. For the $\alpha-\mathrm{SMA}^{+}$area: AP $(n=10)$; APP or normal cecum $(n=7)$. For $\mathrm{Ki}^{+} 7^{+}$colocalization: AP or APP $(n=6)$; normal cecum $(n=4)$. (F) 3D vascular reconstructions from tumor slices. Images show staining for CD31 (green) and E-cadherin (white). Scale bars: $50 \mu \mathrm{m}$. (C) Quantification of vascular parameters and expression of the endothelial marker $C d h 5$. AP $(n=10)$; $\operatorname{APP}(n=7)$; normal cecum $(n=7)$. $\operatorname{AP}$ or $\operatorname{APP}(n=9) ; \mathrm{WT}(n=4)$. Data were normalized to the AP mean. (H) Reduced $C D 8^{+} T$ cell infiltration into APP tumors. Images show staining for $\mathrm{CD}^{+} \mathrm{T}$ cells (green), VE-cadherin (red), and E-cadherin (white). Scale bars: $50 \mu \mathrm{m}$. (I) Quantification of CD8 ${ }^{+}$T cells. AP $(n=10)$; APP $(n=6)$; normal cecum $(n=7)$. (J) APP signature is enriched in PROX1-low human CRCs. Enrichment of the APP versus AP signature in human CRCs (GSE39582; $n=444)$ was computed using $Z$ scores. Dashed line indicates the linear regression fit. Data represent the mean $\pm \mathrm{SD}$. ${ }^{*} P \leq 0.05,{ }^{* *} P \leq 0.01,{ }^{* * *} P \leq$ 0.001 , and ${ }^{* * *} P \leq 0.0001$, by 1-way ANOVA with Tukey's multiple comparisons test (B, E, and $\mathbf{G}$ ) and grouped analysis by 2-way ANOVA with Tukey's multiple comparisons test (E).

Prox1 (Figure 2B and Supplemental Figure 1B), indicating partial inhibition of WNT signaling. Interestingly, the WNT-dependent, cancer-specific V6 isoform of the hyaluronan receptor CD44 (37) was strongly upregulated in APP tumors (Supplemental Figure $1, \mathrm{~B}$ and D). CD44V6 is a marker of CRC tumor-initiating cells that was previously shown to drive CRC invasion and metastasis (38). Unexpectedly, expression of keratin 20 (Krt20), a marker for enterocyte differentiation, was not rescued by Prox1 loss (Figure 2B and Supplemental Figure 1, B and C). Compared with AP cancer cells, APP cancer cells had a decreased proliferation index, as assessed by expression of the proliferation genes Ki67 and cyclin B1 (Ccnb1) (Supplemental Figure 1B). These results suggest that Prox 1 loss in APP tumors skews the expression of WNT-dependent genes and attenuates WNT signaling without inducing differentiation.

Inactivation of Prox1 generates desmoplastic, angiogenic, and $T$ cell-excluded tumors. Comparison of tumor transcriptomes by RNA-Seq analysis revealed significant enrichment of transcripts related to EMT, angiogenesis, myogenesis and oxidative phosphorylation, and downregulation of transcripts related to the adaptive immune response in APP versus AP tumors (Figure 2C). Enrichment in EMT and angiogenesis transcripts is a feature of human desmoplastic CRCs that reflects expansion of the stromal cell compartment $(8,9)$. Staining for $\alpha$-smooth muscle actin ( $\alpha$-SMA), which is expressed in cancer-associated fibroblasts (CAFs) and myofibroblasts, the epithelial marker E-cadherin, and the proliferation marker phosphohistone $\mathrm{H} 3$ ( $\mathrm{PH} 3)$, revealed increased stromal cell content and correspondingly lower epithelial cell content and proliferation in APP compared with AP tumors, indicative of increased desmoplasia (Figure 2, D and E).
Accordingly, mRNA levels of the CAF markers and TGF- $\beta$ target genes fibroblast activation protein $\alpha$ (Fap), periostin (Postn), and serpin family E member 1 (Serpine1, also known as Pai1) (39) were significantly increased (Supplemental Figure 1E). Consistent with the RNA-Seq data, APP tumors displayed a denser and more intricate blood microvascular network, whereas sparse and large blood vessels were observed in AP tumors (Figure 2, F and G). AP tumors displayed areas of high intraepithelial infiltration by $\mathrm{CD}^{+} \mathrm{T}$ cells, which were absent in the majority of APP tumors, indicative of immune exclusion (Figure 2, $\mathrm{H}$ and I).

We computed an enrichment score in human MSS CRCs on the basis of genes differentially expressed in APP versus AP tumors (APP signature) and found that the transcriptomes of the PROX1 $1^{\text {lo }}$ human MSS CRCs were enriched for the APP signature (Figure 2J). Taken together, these results indicate that in mouse intestinal tumors, loss of Prox 1 in cancer cells promotes desmoplasia, angiogenesis, and $\mathrm{T}$ cell exclusion, which are associated with poor prognosis in human CRCs.

Prox1 loss generates desmoplastic tumors in the presence of activated KRAS. In addition to APC and TP53, KRAS is mutated in a significant proportion of CRCs. Constitutive KRAS activation confers an additional growth advantage to APC-mutant cancer cells and promotes resistance to EGFR-targeted therapies in patients with CRC (40). We analyzed the growth of intestinal organoids isolated from Apct/fl $\mathrm{Kras}^{L S L-G 12 D} \mathrm{Tp} 53^{\text {fl/fl }}$ villin-Cre ${ }^{\text {ERT2 }}$ (AKP) and $A p c^{f / f l} K_{r a s}^{L S L-G 12 D} T p 53^{f / f l} P^{2} o x 1^{f / f l}$ villin-Cre ${ }^{E R T 2}$ (AKPP) mice. Prox 1 inactivation reduced the number of organoids that formed in Matrigel from AKPP cells (Figure 3A). These organoids had reduced cell proliferation rates (Supplemental Figure 2A) and grew as large, simple cysts, whereas most AKP organoids formed complex 3D structures (Figure 3B). The analysis of WNT target genes in AKPP organoids showed partial attenuation of WNT signaling but no induction of differentiation (Figure 3C), consistent with APP tumor phenotypes (see above) and our previous findings in KRAS-mutant human CRC cell lines (28). Therefore, Prox1 loss blunted cancer cell-autonomous growth also in the presence of strong KRAS oncogenic signaling.

Prox1-deficient AKPP organoids implanted s.c. displayed rapid outgrowth after a long latency phase, indicating tumor adaptation to PROX1 deficiency (Figure 3D). This result was not due to clonal selection or acquisition of additional mutations, as organoids derived from primary tumors displayed similarly delayed growth kinetics upon ex vivo culturing or secondary implantation in mice (Supplemental Figure 2B). Large primary AKPP tumors (resected on day 93 after implantation) had increased production of the stromal proteins periostin, tenascin $\mathrm{C}$, and $\alpha$-SMA, increased stromal content, and increased expression of the CAF markers and TGF- $\beta$ target genes Fap, Postn, Pail, connective tissue growth factor (Ctgf), and osteopontin (Opn) (Figure 3E and Supplemental Figure 2, C and D), compared with AKP tumors (resected on day 26 after implantation).

Increased accumulation of tumor stroma may be a result of delayed growth of AKPP tumors. To rule out this possibility, we implanted fewer AKP organoids and obtained comparable tumor growth kinetics with AKPP tumors. We then analyzed small sizematched AKP and AKPP tumors, which were resected on days 40 and 48 after implantation, respectively. Even in this setting, 
A

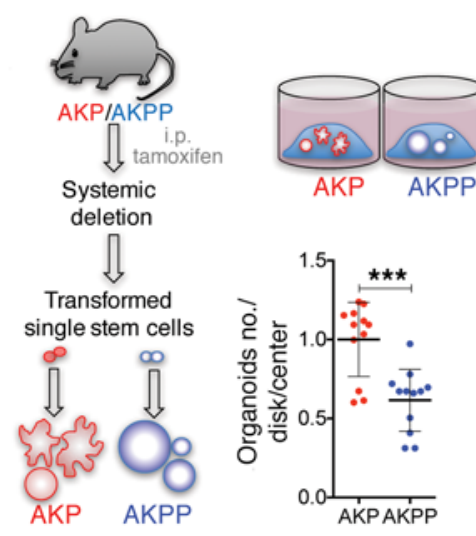

D

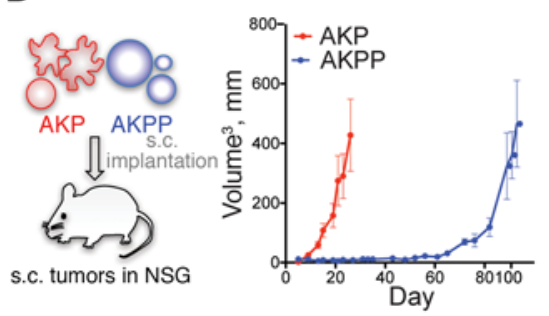

E

Large tumors

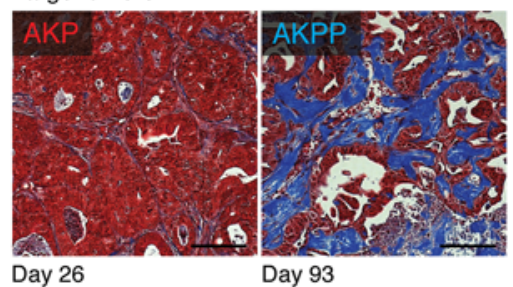

Day 93
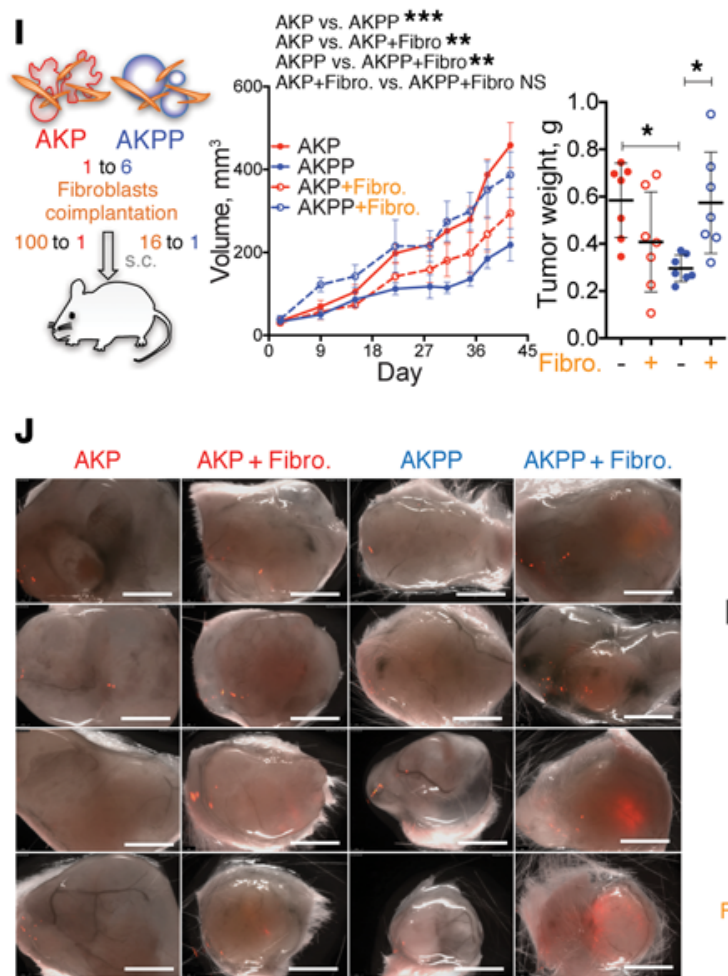

K
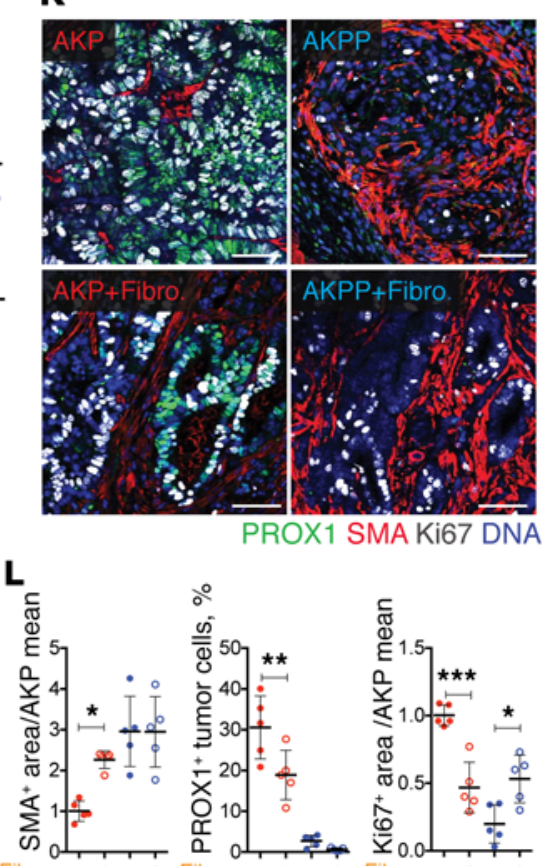

B

$\mathbf{F}$

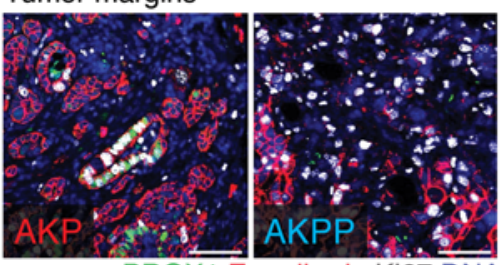

PROX1 E-cadherin Ki67 DNA
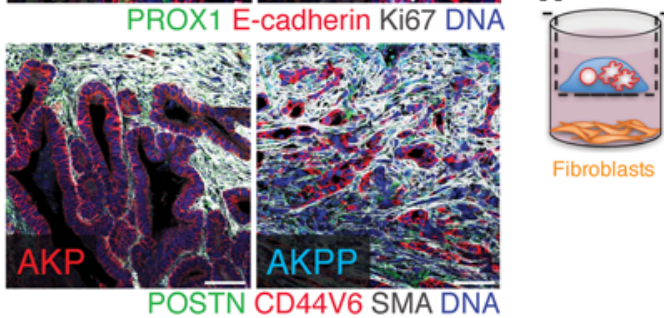

Fibroblasts
G

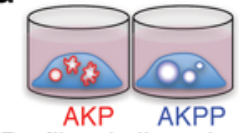

Profibrotic ligands

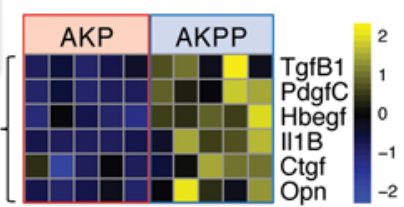

tdTomato fibroblasts
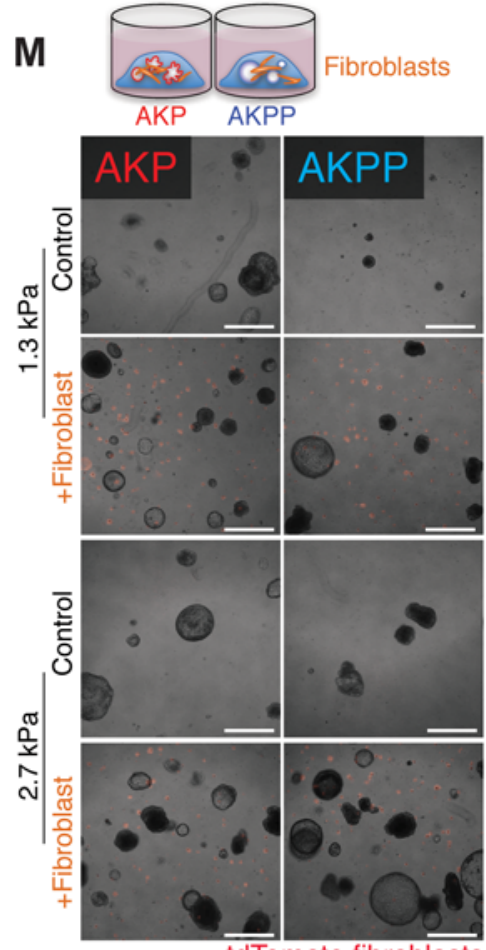

tdTomato fibroblasts 
Figure 3. Prox1 loss generates desmoplastic tumors in the presence of activated Kras. (A) Reduced clonogenic capacity of Prox $1^{-1-}$ intestinal stem

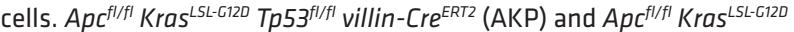
Tp5 $3^{f l / f l}$ Prox ${ }^{f l / f l}$ villin-Cre ${ }^{E R T 2}$ (AKPP) organoids ( $n=12$ per genotype). (B) AKP and AKPP organoids. Upper row shows Matrigel disks with organoids. Scale bars: $1.2 \mathrm{~mm}$. Lower row shows H\&E-stained organoid sections. Scale bars: $20 \mu \mathrm{m}$. Arrow indicates epithelial polarization loss. (C) WNT signaling and differentiation in AKP and AKPP organoids. Graph shows qRT-PCR for the indicated genes, and data are presented in a heatmap $(n=5)$. (D) PROX1 loss delayed tumor development. Mice were injected s.c. with 50 AKP or AKPP organoids. AKP, $n=5$; AKPP, $n=6$. (E) Masson's trichrome staining of advanced tumors. Scale bars: $50 \mu \mathrm{m}$. (F) Increased stromal reaction and invasion in AKPP tumor margins. Images in top row show staining for PROX1 (green), E-cadherin (red), Ki67 (white), and DNA (blue). Images in bottom row show staining for POSTN (green), CD44V6 (red), $\alpha$-SMA (white), and DNA (blue). Scale bars: $50 \mu \mathrm{m}$. (C) AKPP organoids produced profibrotic factors. Heatmap of the indicated genes $(n=$ 5). (H) AKPP organoids promoted fibroblast proliferation. Ki67 and Ccnb1 expression by QRT-PCR. Data were normalized to the control mean $(n=6)$. (I) Tumor growth curves and weights. Mice were implanted with 50 AKP or 300 AKPP organoids \pm 5000 tdTomato $^{+}$intestinal fibroblasts (Fibro.) $(n=7)$. (J) AKPP cells sustained fibroblasts. Images show control and tdTomato (red) fibroblast-coinjected tumors. Scale bars: 2 mm. (K) Fibroblast coimplantation effects on tumor desmoplasia, PROX1 expression, and cell proliferation. Images show staining for PROX1 (green), $\alpha$-SMA (red), Ki67 (white), and DNA (blue). Scale bars: $50 \mu \mathrm{m}$. (L) Quantification of the data shown in $\mathbf{K} . \alpha-\mathrm{SMA}^{+}$and $\mathrm{Ki}^{+} 7^{+}$areas were normalized to the total tumor area and the AKP mean. (M) Fibroblast and tissue stiffness effect on organoids. Organoids with or without tdTomato+ fibroblasts in hydrogels with normal mucosa $(1.3 \mathrm{kPa})$ or tumor $(2.7 \mathrm{kPa})$ stiffness. Scale bars: $1.2 \mathrm{~mm}$. ${ }^{*} P \leq 0.05$, ${ }^{* *} P \leq 0.01$, and ${ }^{* * *} P \leq 0.001$, by Student's $t$ test ( $\mathbf{A}$ and $\mathbf{C}$ ) or 1-way ( $\mathbf{H}$ and $\mathbf{L}$ ) or 2-way (I) ANOVA with Tukey's multiple comparisons test, scatterplot, or mean \pm SD.

AKPP tumors showed increased $\alpha$-SMA ${ }^{+}$stromal cell abundance (Supplemental Figure 2E), indicating that desmoplasia is PROX1 dependent but tumor size independent. The proliferation of cancer cells was lower in AKPP tumors than in AKP tumors, irrespective of tumor size. We examined tumor margins and found a greater number of prominent invading cancer cell strands and increased proliferation of $\alpha-\mathrm{SMA}^{+}$stromal cells in AKPP tumors than in AKP tumors (Figure 3F and Supplemental Figure 2F). Collectively, these data demonstrate that genetic loss of Prox1 generates slow-growing but more invasive and desmoplastic tumors, regardless of the presence of mutant Kras.

Fibroblasts and high tissue stiffness support the growth of Prox1-deficient tumors. Prox1-deficient AKPP organoids expressed higher levels of the profibrotic factors TGF- $\beta 1$ (Tgfb1), plateletderived growth factor $\mathrm{C}(P d g f c)$, heparin-binding EGF-like growth factor (Hbegf), Ctgf, Opn, and IL-1 $\beta$ (Il1b) (Figure 3G), which may contribute to the promotion of expansion and activation of fibroblasts in tumors. Indeed, normal intestinal fibroblasts cocultured with AKPP organoids acquired a CAF-like phenotype characterized by enhanced expression of a-SMA (Acta2), Fap, Postn, Ctgf, and Pail, compared with fibroblasts cocultured with AKP organoids. AKPP-conditioned fibroblasts had greater proliferation, as determined by expression of Ki67 and Ccnb1 (Figure $3 \mathrm{H}$ and Supplemental Figure 2G). Additionally, AKPP organoids also enhanced the expression of $T g f b 1$, matrix metalloproteases, inflammatory cytokines, and proangiogenic Vegfa in fibroblasts (Supplemental Figure 2G).
To determine whether fibroblasts accelerate the progression of Prox1-deficient tumors, we coimplanted AKP or AKPP organoids together with fluorescent intestinal fibroblasts isolated from $\mathrm{mTmG}^{+}$mice, in which all cells express the membrane-targeted tdTomato protein (41). The transplanted fibroblasts significantly enhanced the growth of AKPP but not AKP tumors (Figure 3I). Moreover, the exogenous fibroblasts persisted in AKPP but not AKP tumors, as shown by the presence of tdTomato ${ }^{+}$cells (Figures 3J). Of interest, exogenous fibroblasts enhanced cell proliferation only in the AKPP tumors, as shown by Ki67 staining (Figure 3, K and $\mathrm{L}$, and Supplemental Figure $2 \mathrm{H}$ ). These results support the notion that Prox1-deficient tumors promote a profibrotic TME that in turn sustains the growth of Prox1-deficient cancer cells.

We next examined the effects of intestinal fibroblasts on AKP and AKPP organoids. Because CAF-mediated extracellular matrix (ECM) deposition and increased tumor stiffness promote tumor growth (42), we cultured organoids in synthetic hydrogels with a stiffness similar to that of either normal intestine $(1.3 \mathrm{kPa})$ or CRC stroma (2.7 kPa), as described previously (43), in the presence or absence of fibroblasts. We found that AKPP cells formed smaller organoids in synthetic hydrogels than in Matrigel (0.4 kPa) (Figure 3, $\mathrm{M}$ and $\mathrm{B}$ above). However, the addition of fibroblasts or high stiffness partly rescued, and their combination fully restored, the growth of AKPP organoids (Figure 3M and Supplemental Figure 2I). Conversely, fibroblasts and stiffness had modest, if any, effects on the growth of AKP organoids. Thus, both fibroblastderived factors and increased tumor stiffness probably contribute to the promotion of AKPP tumor growth.

Chemotherapy promotes stromal activation and progression of Prox1-deficient tumors. 5-FU-based adjuvant chemotherapy is the standard of care for CRC and improves patient survival; however, a substantial proportion of the patients either do not respond to this treatment or relapse (2). To determine whether PROX1 regulates responses to chemotherapy, we tested 5-FU or cisplatin as a control agent in AKP and AKPP organoids. Prox1-deficient AKPP organoids were less sensitive to 5-FU, an antimetabolite, than were AKP organoids, whereas AKP and AKPP organoids were similarly sensitive to cisplatin, which acts via a DNA cross-linking mechanism (Supplemental Figure 3A).

We next investigated the response of s.c. AKP and AKPP tumors to chemotherapy. In order to obtain AKP and AKPP tumors with comparable growth rates, we injected an excess of AKPP organoids and initiated the treatment when tumors reached approximately $130 \mathrm{~mm}^{3}$ in size (Figure $4 \mathrm{~A}$ ). Both 5 -FU and cisplatin rapidly arrested the growth of AKP tumors, however, they did not affect AKPP tumors. At the end of the experiments, the weight of treated AKP tumors was significantly reduced, whereas no difference was observed between the control and treated AKPP tumors (Figure 4A). Microscopic analysis showed that chemotherapy inhibited cancer cell proliferation in the core of AKP tumors (Supplemental Figure 3B), leading to significant central necrosis, but we observed no such effects in AKPP tumors, which had low cancer cell proliferation and high necrosis at baseline (Supplemental Figure 3B). We observed major differences in the tumor margins. Chemotherapy inhibited cancer cell proliferation and reduced peritumoral vascular density at the margins of the AKP tumors, whereas the AKPP tumor margins 
A

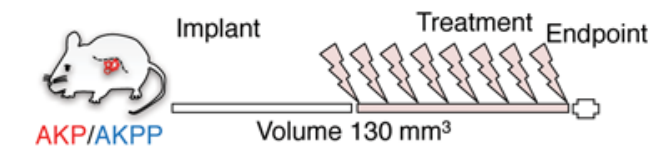
tumors in NSG
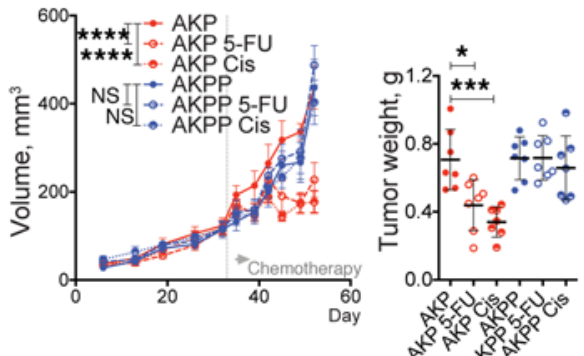

D
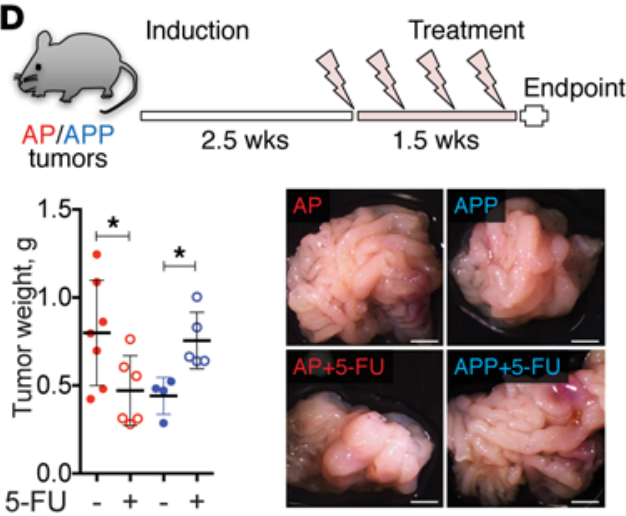

$\mathbf{F}$
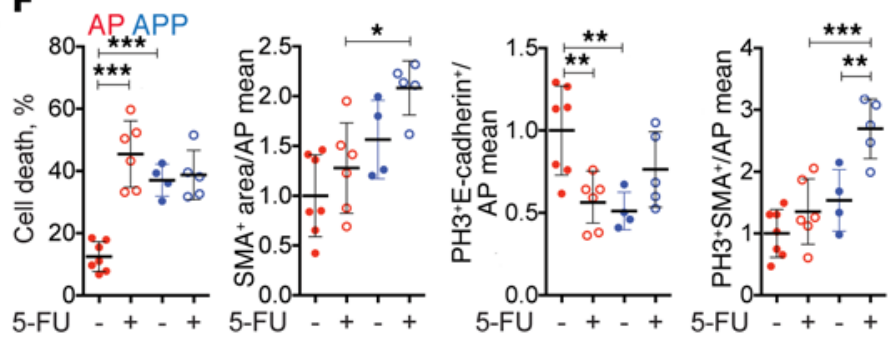

G

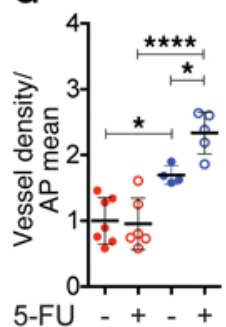

C

Tumor margins AKP AKPP

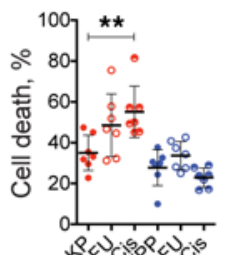

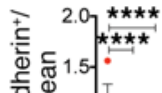

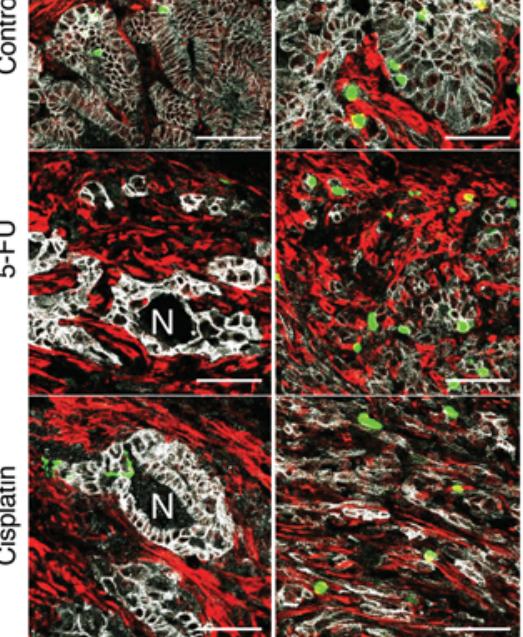

PH3 SMAE-cadherin

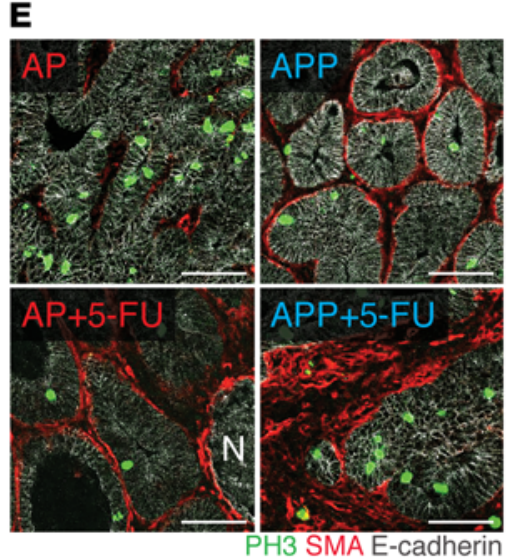

H
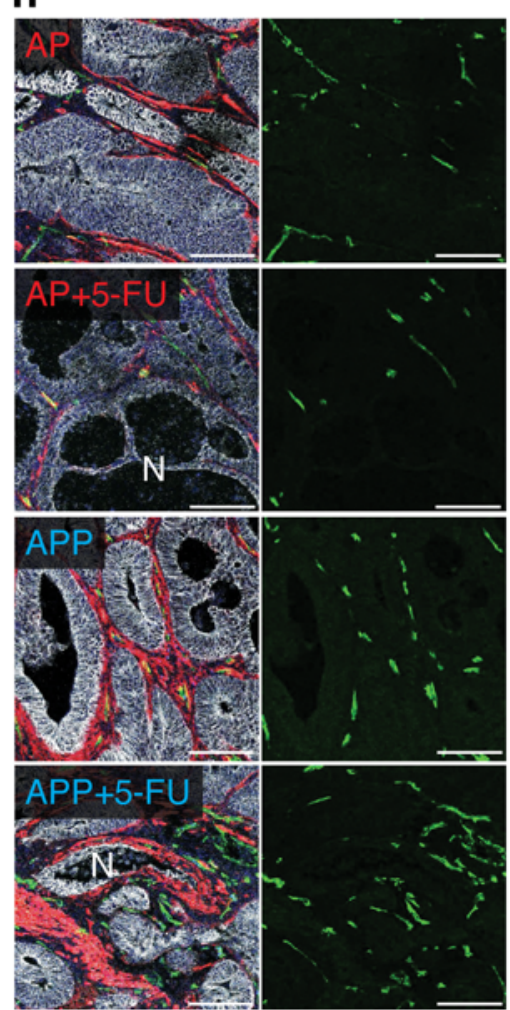

CD31 SMA E-cadherin DNA

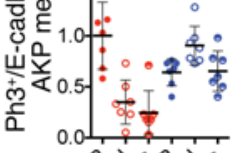

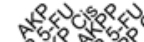
bs

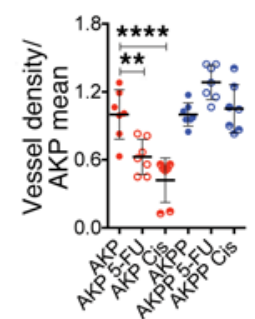

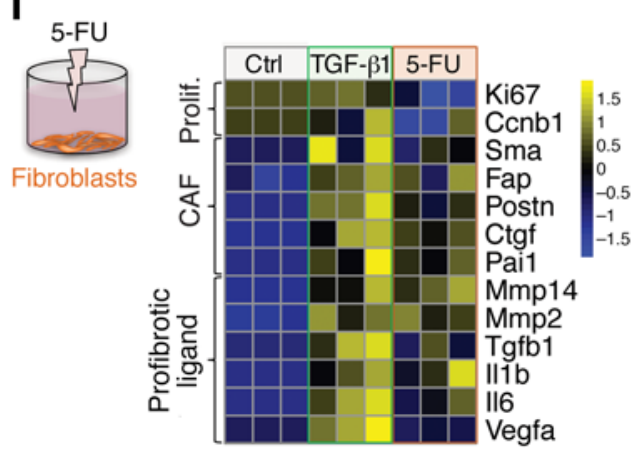


Figure 4. Prox1-deficient tumors are chemoresistant. (A) Tumor response to chemotherapy. Mice were implanted s.c. with 50 AKP or 300 AKPP organoids, and treatment with 5-FU or cisplatin was initiated when the mean volume of the tumors reached $130 \mathrm{~mm}^{3}(n=7)$. (B) Chemotherapeutic effect on tumor margins. Images show staining for PH3 (green), $\alpha-5 M A$ (red), and E-cadherin (white). Scale bars: $50 \mu \mathrm{m}$. (C) Quantification of cell death, proliferation, and angiogenesis for the images in B. Cell death is indicated as the percentage of necrotic tumor glands. Other data are indicated by the fold change versus the AKP control mean. Cis, cisplatin. (D) Macroscopic appearance of control- and 5-FU-treated AP and APP tumors and tumor weights at sacrifice. AP control, $n=7$; AP 5-FU, $n=6$; APP control, $n=4$; APP 5-FU, $n=5$. Scale bars: $2 \mathrm{~mm}$. (E) 5-FU effects on tumor desmoplasia and cancer cells. Images show staining for PH3 (proliferation, green), $\alpha$-SMA (fibroblasts, red), and E-cadherin (tumor cells, white). Scale bars: $50 \mu \mathrm{m}$. (F) Quantification of tumor cell proliferation and death. $\alpha-\mathrm{SMA}^{+}, \mathrm{PH}^{+} \mathrm{E}-$ cadherin ${ }^{+}$, and $\mathrm{PH}^{+} \alpha-\mathrm{SMA}^{+}$areas were quantified and normalized to total tumor E-cadherin ${ }^{+}$or $\alpha-\mathrm{SMA}^{+}$areas and the AP control mean. Cell death is shown as a percentage of necrotic tumor glands. (G) 5-FU effect on angiogenesis. CD31+ vessel density is presented as the fold change versus the AP control mean. (H) Images show staining for CD31 (green), $\alpha$-SMA (red), E-cadherin (white), and DNA (blue). Scale bars: $50 \mu \mathrm{m}$. (I) 5-FU suppressed proliferation and promoted the expression of CAF markers and fibrotic ligand in cultured fibroblasts. A heatmap of the indicated genes is shown. Intestinal fibroblasts were cultured for 24 hours in the presence of $2.5 \mu \mathrm{M} 5$-FU, $50 \mathrm{ng} / \mathrm{mL}$ TCF- $\beta 1$, or control $(n=3)$. Data represent the mean $\pm \mathrm{SD}$. ${ }^{*} P \leq 0.05,{ }^{* *} P \leq 0.01,{ }^{* *} P \leq 0.001$, and ${ }^{* * *} P$ $\leq 0.001$, by 1-way (scatterplots) or 2-way (growth curves) ANOVA with Tukey's multiple comparisons test. N, necrosis.

showed high fibroblast proliferation, sustained vascularization, and exacerbated invasion (Figure 4, B and C, and Supplemental Figure 3C), with occasional intravasation of cancer cells into peritumoral blood vessels (Supplemental Figure 3D), which we never observed in AKP tumors. Together, these results suggest that loss of Prox1 protects tumors from the effects of chemotherapy.

We next studied the effects of chemotherapy on autochthonous (tamoxifen-induced) AP and APP tumors. 5-FU significantly reduced AP tumor size, however, it unexpectedly enhanced the growth of APP tumors (Figure 4D). In analogy with observations in the s.c. models, 5-FU induced cell death and reduced cancer cell proliferation in AP but not APP tumors (Figure 4, E and F). Importantly, both fibroblast proliferation and angiogenesis were further increased in 5-FU-treated APP tumors (Figure 4, F-H). In addition to CAF and angiogenesis markers, we found that $I l 1 b$ and $L y 6 g$ were also induced, indicating increased inflammation and an influx of neutrophils (Supplemental Figure 3E).

Previous studies demonstrated that 5-FU increases TGF- $\beta$ release and signaling (44). We then compared the effects of TGF- $\beta 1$ or a clinically relevant $(2.5 \mu \mathrm{m})$ of 5 -FU (45) on cultured intestinal fibroblasts. We observed that 5-FU reduced fibroblast proliferation but promoted the expression of $T g f b 1$, as well as several TGF- $\beta$ target genes such as Pail, Fap, and Acta2 ( $\alpha$-SMA) (Figure 4I). The effects of 5-FU on stromal cell activation were comparable to those of TGF- $\beta 1.5$-FU also enhanced the expression of the proangiogenic and proinflammatory cytokines Vegfa, Illb, and $I l 6$. IL-6 is an upstream regulator of JAK/STAT3 signaling, which was previously shown to sustain colon cancer cell survival and WNT activation $(46,47)$. In the tumors, 5-FU did not alter the expression of WNT target genes (Supplemental Figure 3F), but both cancer and stromal cells in 5-FU-treated APP tumors displayed increased nuclear accumulation of phosphorylated STAT3 (p-STAT3) (Supplemental Figure 3G). In summary, our results in both s.c. and autochthonous tumor models indicate that chemotherapy is highly effective in $\mathrm{WNT}^{\text {hi }} \mathrm{PROX} \mathrm{1}^{\text {hi }}$ tumors. However, chemotherapy promotes TGF- $\beta$-dependent fibroblast activation and production of angiogenic and protumoral factors in PROX $1^{10}$ desmoplastic tumors and is thus detrimental to the outcome.

The PROX1 target gene Mmp14 induces tumor fibrosis and chemoresistance. $\mathrm{PROX} 1$ is a transcriptional repressor shown previously to directly repress annexin A1 (ANXA1) in Apc-deficient mouse intestinal stem cells (29) and matrix metalloproteinase 14 (MMP14) in human liver, colon, and breast cancer cell lines (48). Accordingly, the expression of both genes was increased in cultured Prox1deficient organoids and tumors (Figure 5A). In human CRC subtypes, the highest MMP14 levels were observed in desmoplastic CMS4 tumors, whereas ANXA1 was prominent in both CMS1 and CMS4 tumors (Figure 5B). 5-FU further enhanced the expression of Mmp14 but not Anxa1 in Prox1-deficient organoids and tumors, along with expression of the profibrotic factors Tgfb1, Pdgfc, Hbegf, and $I l 1 b$ (Figure 5C and Supplemental Figure 4, A and B).

To investigate whether ANXA1 or MMP14 were implicated in inducing the desmoplastic phenotype of Prox1-deficient tumors, we generated Anxa1- or Mmp14-overexpressing AKP organoids (ANXA1-AKP and MMP14-AKP, respectively) and, as a control, organoids expressing a nonsignaling form of the low-affinity nerve growth factor receptor (dlNGFR-AKP) (Supplemental Figure 4C). Overexpression of either protein did not affect the growth of organoids in vitro or the expression of WNT target genes (Supplemental Figure 4C). Upon s.c. injection, ANXA1-AKP and control dlNGFR-AKP tumors had similar growth rates, however, MMP14AKP tumors grew at a significantly slower rate (Figure 5D). Histological analysis revealed that overexpression of MMP14 was associated with stromal cell proliferation and expansion, prominent angiogenic response, and reduced cancer cell proliferation (Figure 5, E and F, and Supplemental Figure 4D). These results were further corroborated by mRNA analysis of stromal and angiogenesis markers (Supplemental Figure 4E). MMP14-AKP tumors lacked the central necrosis observed in AKPP tumors and displayed no change in WNT signaling (Supplemental Figure 4E), indicating partial phenocopying of Prox1-deficient tumors.

We next examined whether MMP14 overexpression was sufficient to confer tumor resistance to chemotherapy. 5-FU arrested the growth of control tumors but had no inhibitory effects on MMP14-AKP tumors (Figure 5G). Similar to the results in AKPP tumors, 5-FU did not affect cancer cell proliferation and further increased fibrosis and angiogenesis in MMP14-AKP tumors (Figure 5, H-J, and Supplemental Figure 4F). We also observed that transgenic MMP14 in cancer cells induced endogenous MMP14 expression in stromal cells, an effect that was heightened by chemotherapy (Figure 5H and Supplemental Figure 4G). Together, these results suggest that tumor desmoplasia, angiogenesis, and resistance to chemotherapy induced by loss of Prox 1 are mediated through the derepression of its direct target MMP14.

Reprogramming the angiogenic and immune microenvironment restrains the growth of chemoresistant Prox1-deficient tumors. The aforementioned data indicate that chemoresistance of APP tumors is associated with a highly angiogenic and T cell-excluded 
A

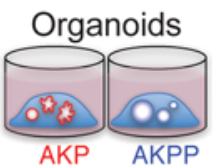

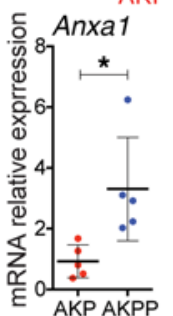

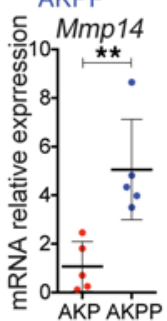

Tumors

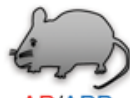

APIAPP
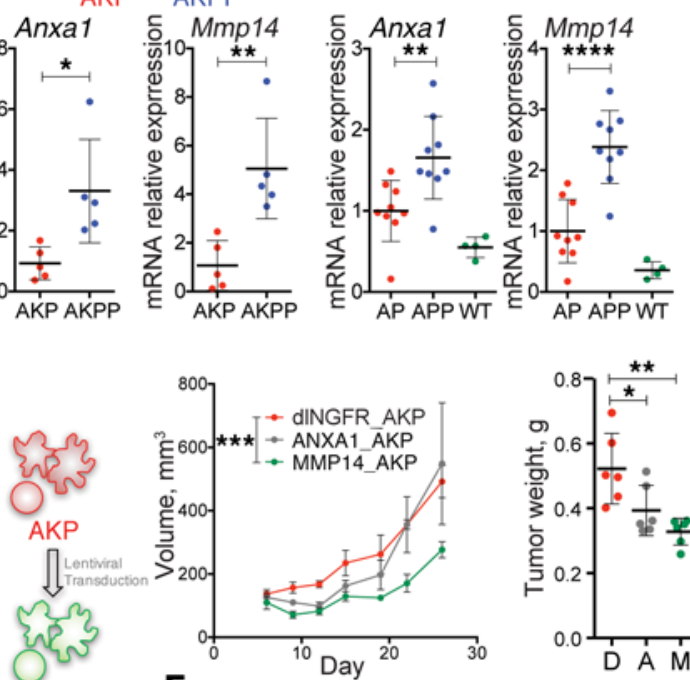

PROX1targets overexpression<smiles>O=C1C=CC=CC1</smiles>

Tumors in NSG

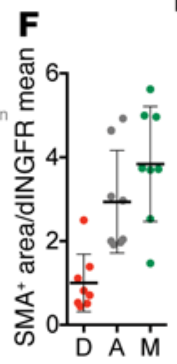

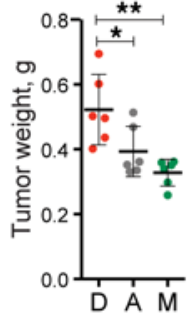

D A M
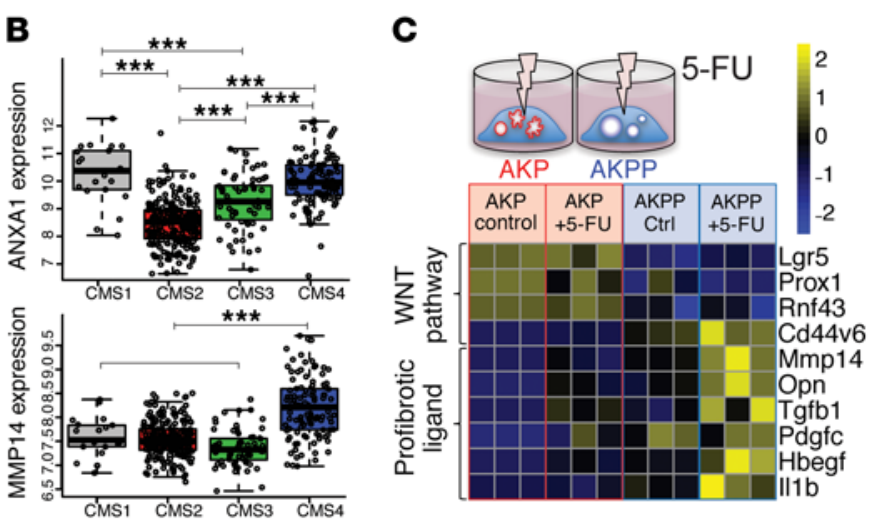

E

AKP. $\left\{\begin{array}{l}\text { dINGFR control } \\ \text { Annexin A1 }\end{array}\right.$

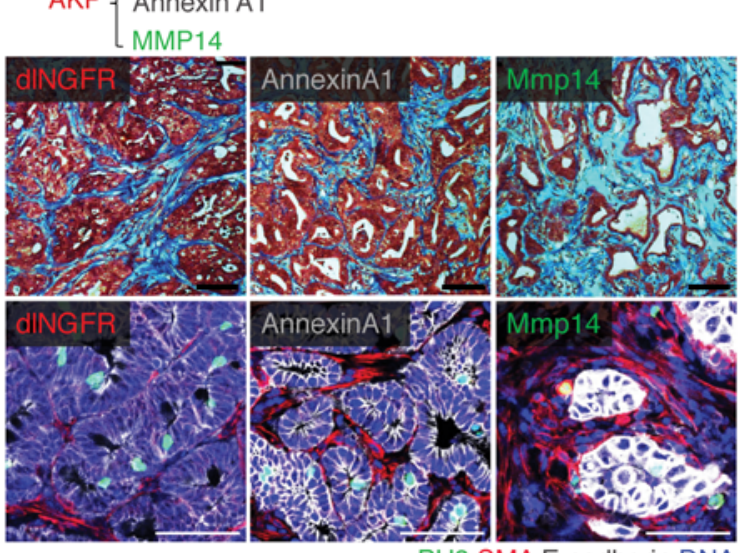

PH3 SMA E-cadherin DNA

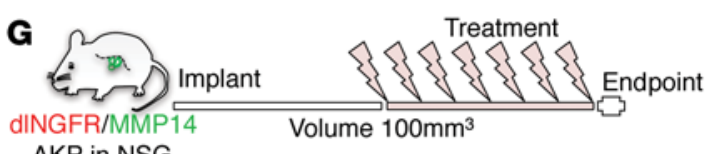
AKP in NSG

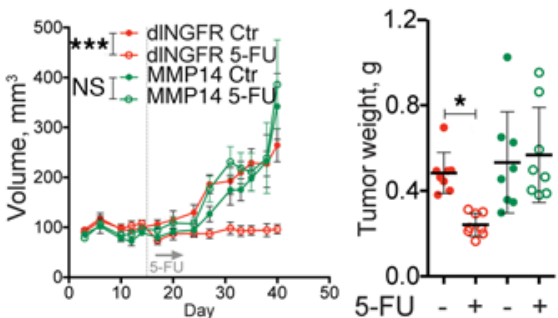

H
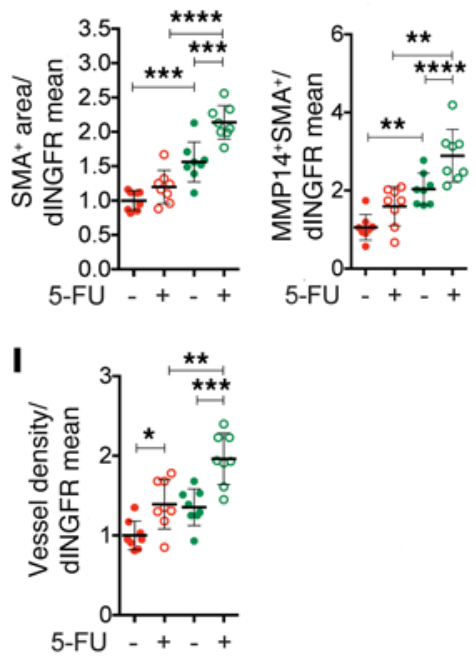

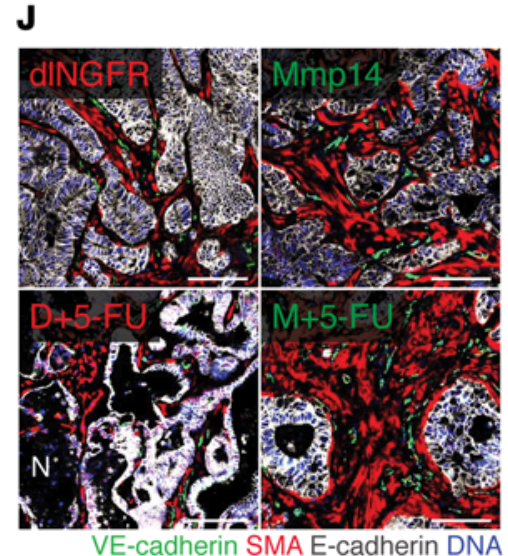


Figure 5. The PROX1 target MMP14 recapitulates tumor desmoplasia, angiogenesis, and chemoresistance. (A) Mmp14 and Anxa1 expression in organoids and tumors. qRT-PCR data were normalized to the AKP organoids or the mean of AP tumors. (B) MMP14 and ANXA1 expression in CRC CMS classification (GSE39582; $n=409$ ). $P<0.001$, by 1-way ANOVA with Tukey's multiple comparisons test. (C) 5-FU effect on WNT targets and profibrotic factors. A heatmap of the indicated genes is shown $(n=3)$. (D) Tumor growth and weights after overexpression of MMP14 or ANXA1. Fifty MMP14-AKP, ANXA1-AKP, or control dINGFR-AKP organoids were implanted s.c. per mouse $(n=6)$. (E) MMP14 overexpression promoted desmoplasia. Images in top row show Masson's trichrome staining; images in bottom row show staining for PH3 (green), $\alpha$-SMA (red), E-cadherin (white), and DNA (blue). Scale bars: $50 \mu \mathrm{m}$. (F) Quantification of stromal content, stromal and cancer cell proliferation, and vascular density in control and MMP14- or ANXA1-overexpressing tumors. Data are presented as the fold change versus the AKP control mean or versus the MMP14 mean for stromal proliferation. (G) MMP14-AKP tumors were chemoresistant. Mice were implanted s.c. with 50 control dINGFR-AKP or 100 MMP14-AKP organoids $(n=8) .5$-FU treatment was started when tumors reached $100 \mathrm{~mm}^{3}$ in size. (H) 5-FU effect on desmoplasia and stromal MMP14 expression in MMP14AKP tumors. Quantification of $\alpha-\mathrm{SMA}^{+}$stromal area and $\mathrm{MMP}^{+} 4^{+} \alpha-\mathrm{SMA}^{+}$ coexpression in control dINGFR-AKP and MMP14-AKP tumors. Data are presented as the fold change versus the AKP control mean. (I) 5-FU effect on angiogenesis in MMP14-AKP tumors. VE-cadherin ${ }^{+}$vessel density is presented as the fold change versus the AKP control mean. (J) Effect of 5-FU on angiogenesis in MMP14-AKP tumors. Images show staining for VE-cadherin (green), $\alpha$-SMA (red), E-cadherin (white), and DNA (blue). Scale bars: $50 \mu \mathrm{m}$. Data represent the mean $\pm \mathrm{SD}$. ${ }^{*} P \leq 0.05,{ }^{*} P \leq 0.01$, ${ }^{* * *} P \leq 0.001$, and ${ }^{* * *} P \leq 0.0001$, by Student's $t$ test $(\mathbf{A})$ or 1 -way ANOVA (scatterplots) or 2-way ANOVA (growth curves) with Tukey's multiple comparisons test. D, dINGFR; A, Annexin A1; M, MMP14.

desmoplastic microenvironment. This prompted us to pursue reprogramming of the TME as an alternative therapeutic strategy. Previous studies have shown that anticancer immunity can be efficiently triggered through the activation of CD40 in desmoplastic pancreatic ductal adenocarcinoma $(20,21)$. We then used a murinized version of A2V to block both VEGFA and ANGPT2 (49) (17) in combination with FGK4.5, an agonistic CD40 (aCD40) antibody (50), in genetic models of CRC. We started the treatments 2 weeks after tumor initiation and terminated the experiments 2 weeks later (Figure 6A). Treatment with aCD40 had a minor, if any, effect on tumor growth in either model, whereas $\mathrm{A} 2 \mathrm{~V}$ had a measurable growth-inhibitory effect on AP but not APP tumors. In contrast, the combination of $\mathrm{A} 2 \mathrm{~V}$ and aCD40 inhibited the growth of both AP and APP tumors (Figure 6A). Further microscopic analysis revealed cancer cell death and an accumulation of $\mathrm{p}-\gamma \mathrm{H} 2 \mathrm{~A} . \mathrm{X}^{+}$ nuclear foci specifically in tumors treated with the A2V+aCD40 combination (Figure 6, B and C, and Supplemental Figure 5, A and B). Similarly, cancer cell proliferation was significantly reduced only by the combination treatment in both models (Figures 6C). Together, these results demonstrate that the combination of $\mathrm{A} 2 \mathrm{~V}$ and aCD40 had marked antitumoral activity in both AP and chemoresistant APP models.

Combined $A 2 \mathrm{~V}$ and aCD4O antibody therapy normalizes tumor blood vessels and reduces fibrosis. We next examined the effects of treatments on tumoral vascular density, vessel caliber, mural cell coverage, tumor hypoxia, and CAF activation and proliferation. A2V did not alter the relatively sparse vascular network of AP tumors, whereas it pruned the angiogenic vasculature of APP tumors (Figure 6, D and E). In both tumor models, A2V and the combined treatment, but not aCD40 monotherapy, enhanced blood vessel coverage by $\alpha-\mathrm{SMA}^{+}$mural cells and increased the caliber of the remaining blood vessels. To evaluate vascular functionality, we analyzed tumor hypoxia by staining for pimonidazole adducts. We found low levels of hypoxia in control AP and APP tumors, which were not modified by the treatment (Supplemental Figure 5, C and D). These data indicate that AP and APP tumors were well perfused at baseline and that the main effect of $\mathrm{A} 2 \mathrm{~V}$ in these models was the promotion of vascular maturation. In APP tumors, aCD40 monotherapy did not obviously alter the tumor stroma, whereas $\mathrm{A} 2 \mathrm{~V}$ reduced stromal cell content and the expression of the activation marker $\alpha$-SMA in CAFs and myofibroblasts (Figure 6, B and F) as well as of the CAF markers and TGF- $\beta$ target genes Fap, Ctgf, and Pai1 (Supplemental Figure 6A), indicating that $\mathrm{A} 2 \mathrm{~V}$ limits stromal activation in this model.

$\mathrm{A} 2 \mathrm{~V}+\mathrm{aCD} 40$ also downregulated Mmp14 expression and promoted even greater destruction of the stroma, compared with $\mathrm{A} 2 \mathrm{~V}$ monotherapy in the desmoplastic APP tumors, as shown by both reduced stromal cell proliferation and content (Figure 6, B and G). Altogether, these results demonstrate that dual blockade of VEGFA and ANGPT2, especially in combination with aCD40, normalizes tumor blood vessels and reduces tumor fibrosis.

Combined $\mathrm{A} 2 \mathrm{~V}$ and aCD4O antibody therapy promotes intraepithelial infiltration of cytotoxic $C D 8^{+} T$ cells. We next analyzed tumor-infiltrating lymphocytes (TILs). Although neither monotherapy had significant effects on the total number of intratumoral $\mathrm{CD}^{+}$or $\mathrm{CD}^{+} \mathrm{T}$ cells (Supplemental Figure $6 \mathrm{~B}$ ), we found that $\mathrm{A} 2 \mathrm{~V}+\mathrm{aCD} 40$ promoted the influx of $\mathrm{CD}^{+} \mathrm{T}$ cells and increased the CD8/CD4 ratio in APP tumors (Figure 6, H and I, and Supplemental Figure 6B).

Engagement of $\mathrm{CD}^{+} \mathrm{T}$ cells with epithelial cells is strongly associated with a favorable prognosis in CRC (51). We therefore characterized the spatial distribution of $\mathrm{T}$ cells within tumors. $\mathrm{A} 2 \mathrm{~V}+\mathrm{aCD} 40$ markedly induced intraepithelial infiltration of $\mathrm{CD}^{+} \mathrm{T}$ cells into both AP and APP tumors (Figure 6, H and I). Most important, the combined treatment dramatically increased the proportion of granzyme $\mathrm{B}^{+}\left(\mathrm{GZMB}^{+}\right) \mathrm{CD}^{+} \mathrm{T}$ cells, which is indicative of an activated cytotoxic phenotype, in APP tumors; a similar but less marked response was observed in AP tumors (Figures 6J and Supplemental Figure 6C). Tregs suppress effector T cells, and a high $\mathrm{CD}^{+} /$Treg cell ratio is generally associated with a favorable prognosis in solid cancers (52). In both tumor models, A2V strongly reduced the abundance of intratumoral $\mathrm{CD} 4^{+} \mathrm{Foxp} 3^{+}$ Tregs, resulting in a significantly higher $\mathrm{CD} 8^{+} /$Treg ratio, an effect that was maintained in the $\mathrm{A} 2 \mathrm{~V}+\mathrm{aCD} 40$ group (Figure 6, $\mathrm{K}$ and $\mathrm{L}$ ).

We further analyzed genome-wide transcriptional responses of tumors to $\mathrm{A} 2 \mathrm{~V}+\mathrm{aCD} 40$ treatment using gene set enrichment analysis (GSEA) (53). The most prominent pathways activated by $\mathrm{A} 2 \mathrm{~V}+\mathrm{aCD} 40$ in AP and APP tumors were related to allograft rejection and IFN signaling (Figure $6 \mathrm{M}$ ), thus confirming the ability of $\mathrm{A} 2 \mathrm{~V}+\mathrm{aCD} 40$ to convert the immunologically "cold-phenotype" AP and APP tumors into a highly inflamed state that was potentially conducive to an efficient anticancer immune response. Surprisingly, using signatures for mouse immune cell populations (54), we found that, together with the expected increase in DC and $\mathrm{T}$ cell signatures, $\mathrm{A} 2 \mathrm{~V}+\mathrm{aCD} 40$ treatment also led to enrichment of B cell-associated transcripts, whereas the macrophage 
A

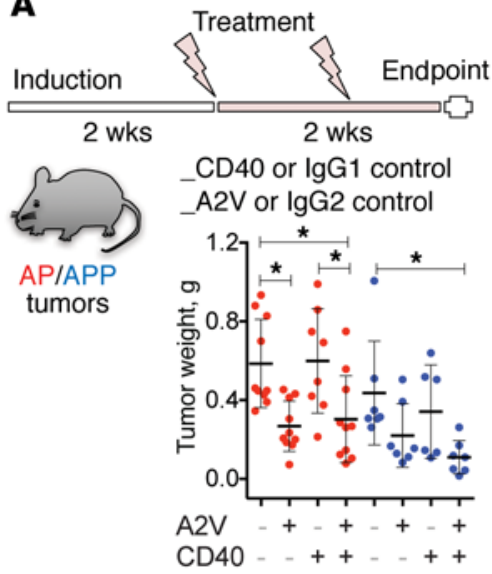

D

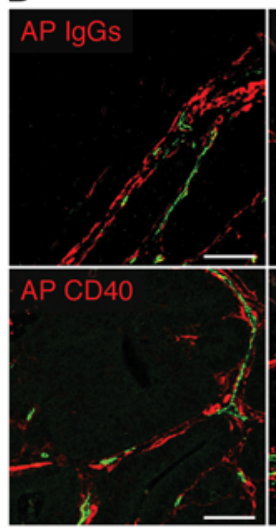

$\mathbf{F}$

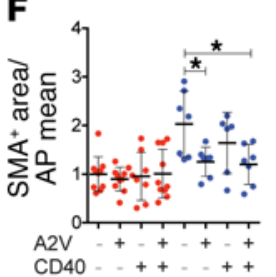

G
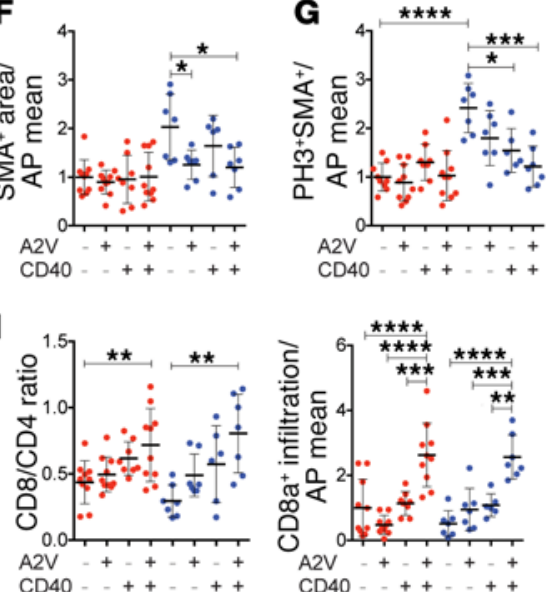

K

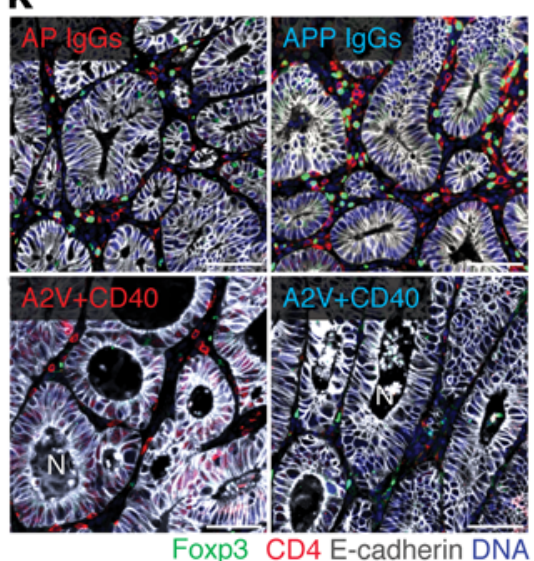

B

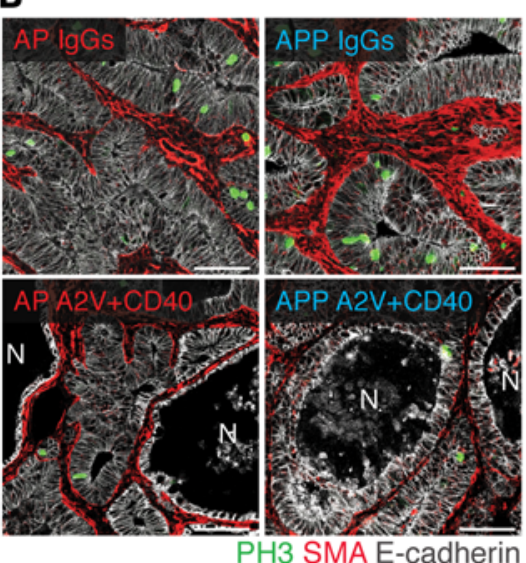

PH3 SMA E-cadherin

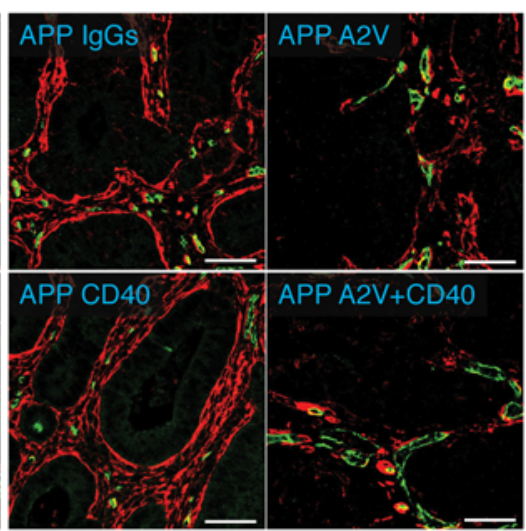

CD31 SMA
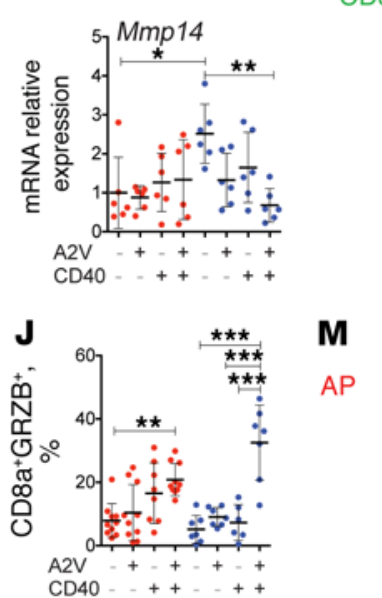

$\mathbf{L}$
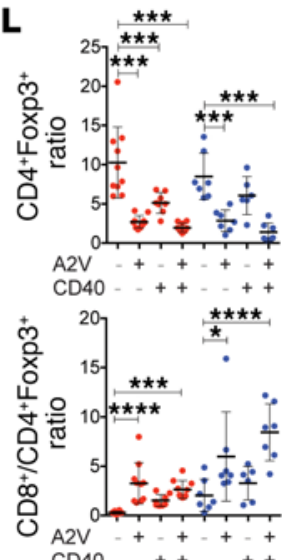

APP

$$
\text { PP }
$$
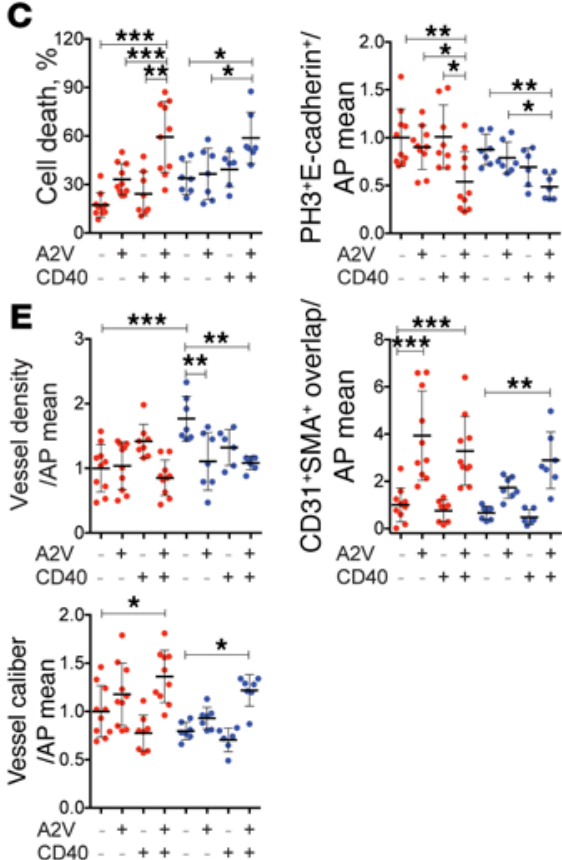

H
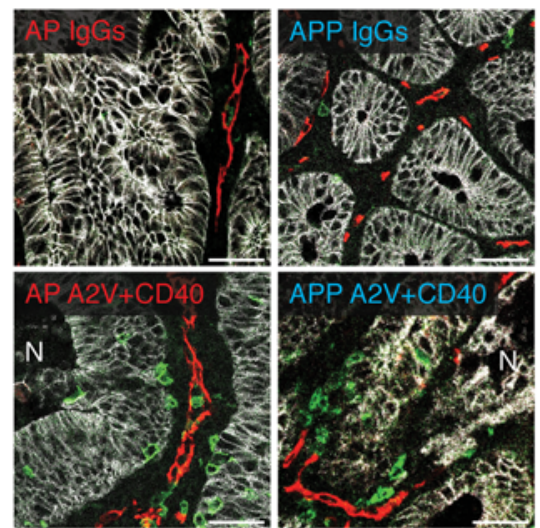

CD8a VE-cadherin E-cadherin

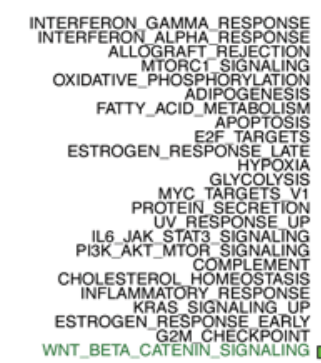

NES

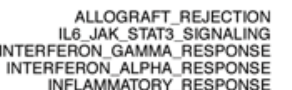

TERFERON ALPHA RESPONSE
INFLAMMATORY RESPONSE

NFA_SIGNALING VIA NFK

KRAS SIGNALING UP

XENOBIOTICOAGULATION

EPITHELIAL MESENCHYMAL APOPTOSIS

P53 PATHWAY
UNFOLDED PROTEIN RESPONS
OXIDATIVE PHOSPHOPYTION

OXIDATIVE. PHOSPHORYLATIO
WNT BETA CATENIN SIGNALING

G2M CHECKPOIN

NES

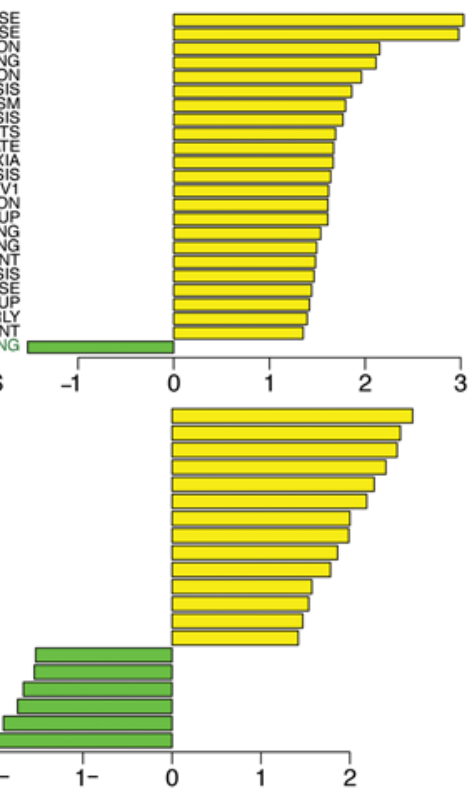


Figure 6. A2V+aCD40 combination reduces AP and APP tumor growth.

(A) Treatment scheme and weights of tumors in the indicated conditions. AP IgGs, AP A2V, or AP A2V+aCD40, $n=10$; AP aCD40, $n=8$; APP IgGs, APP A2V or APP A2V $+a C D 40, n=7$; APP aCD40, $n=6$. (B) A2V+aCD40 induced tumor necrosis and stromal destruction. Images show staining for E-cadherin (white), PH3 (green), and $\alpha$-SMA (red). Scale bars: $50 \mu \mathrm{m}$. (C) Quantification of tumor necrosis and proliferation. Necrosis is indicated by the percentage of necrotic glands. Proliferation was quantified by $\mathrm{PH}^{+}{ }^{+}$-cadherin ${ }^{+}$tumor cells normalized to total $\mathrm{E}$-cadherin ${ }^{+}$and the AP IgGs mean. (D) A2V and A2V+aCD40 promoted vascular maturation. Images show staining for CD31 (green) and $\alpha$-SMA (red). Scale bars: $50 \mu \mathrm{m}$. (E) Quantification of vessel density, mural coverage, and vessel caliber. Data were normalized to the AP IgGs mean. (F) A2V and A2V+aCD40 reduced desmoplasia. The $\alpha-S M A^{+}$stromal area was normalized to the corresponding tumor area and the AP IgGs mean. (G) A2V and A2V+aCD40 effects on stromal proliferation and Mmp14 mRNA. Quantification of $\mathrm{PH}^{+} \alpha-S M A^{+}$ fibroblasts per tumor area and qRT-PCR for Mmp14 in tumor lysates. Data were normalized to the AP IgGs mean. $(\mathrm{H}) \mathrm{A} 2 \mathrm{~V}+\mathrm{aCD} 40$ promoted $C D 8^{+} \mathrm{T}$ cells intraepithelial infiltration. Images show staining for CD8a (green), VE-cadherin (red), and E-cadherin (white). Scale bars: $50 \mu \mathrm{m}$. (I) CD8 ${ }^{+} / \mathrm{CD}^{+}$ TIL ratio and intraepithelial $C D 8^{+} T$ cell quantification were determined as the fold change versus the AP IgGs mean. (J) A2V+aCD40 increased GZMB ${ }^{+}$ cytotoxic $C D 8^{+} T$ cells. Intratumoral $C D 8^{+} C R Z B^{+} T$ cells were normalized to $C D 8^{+} \mathrm{T}$ cells and the AP IgGs mean. (K) A2V and A2V+aCD40 reduced Tregs. Images show staining for Foxp3 (green), CD4 (red), and E-cadherin (white). Scale bars: $50 \mu \mathrm{m}$. (L) Quantification of Tregs and $\mathrm{CD} 8^{+} /$Treg ratio. $\mathrm{CD}^{+}{ }^{+}$oxp3 $^{+}$expression was normalized to $\mathrm{CD}^{+} \mathrm{T}$ cells and the AP IgGs mean. (M) Pathways deregulated by A2V+aCD40, AP A2V+aCD40, or APP $\mathrm{A} 2 \mathrm{~V}+\mathrm{aCD} 40, n=4$; AP or APP controls, $n=6$. FDR $<0.05$. NES, normalized enrichment scores. Data represent the mean $\pm \mathrm{SD}$. ${ }^{*} P \leq 0.05$, ${ }^{* *} P \leq 0.01$, ${ }^{* * *} P \leq 0.001$, and ${ }^{* * *} P \leq 0.0001$, by 1 -way ANOVA with Tukey's multiple comparisons test.

signature was not affected (Supplemental Figure 6D). Therefore, we analyzed the distribution of intratumoral B cells by staining B220 in control (IgG-treated) tumors compared with treated tumors. We observed few and sparse B cells in both control- and aCD40-treated tumors. Interestingly, we found an increased incidence of large peri- and intratumoral B cell clusters in $\mathrm{A} 2 \mathrm{~V}$ - and A2V+aCD40-treated tumors (Supplemental Figure 6, E-G). Such clusters harbored both $\mathrm{B}$ and $\mathrm{T}$ cells and contained HEV-like vessels lined with plump ECs (Supplemental Figure 6G). These results demonstrate that $\mathrm{A} 2 \mathrm{~V}$ promoted the generation of tertiary lymphoid structures (TLSs), which are nonencapsulated immune cell aggregates that are associated with productive antitumor immunity and a favorable prognosis in many cancer types, including CRC (55). Taken together, our results demonstrate that A2V profoundly reprogrammed the TME in mouse models of CRC and potentiated immune activation in response to aCD40 signaling.

The antitumoral activity of $A 2 V+a C D 4 O$ is $T$ cell and ANGPT2 dependent. Agonistic CD40 antibodies activate antigen-presenting cells and promote antitumoral, $\mathrm{T}$ cell-dependent responses. They also induce tumoricidal macrophages that were shown to control tumors by promoting tumor stroma destruction in the absence of $\mathrm{T}$ cell immunity $(20,56,57)$. We then asked whether the antitumoral activity of $\mathrm{A} 2 \mathrm{~V}+\mathrm{aCD} 40$ is $\mathrm{T}$ cell dependent. To this aim, we induced concomitant depletion of $\mathrm{T}$ cells using $\alpha$-CD8a and $\alpha$-CD 4 antibodies (Figure 7A). We achieved efficient $\mathrm{T}$ cell ablation from tumors, as determined by staining of tumor sections with noncompeting $\alpha-\mathrm{CD} 8$ and $\alpha-\mathrm{CD} 4$ antibodies, or by FACS analysis of draining lymph nodes using $\alpha$-CD3 (Figures 7A and Supplemental Figure 7A). Because A2V+aCD40 in combination with $\mathrm{T}$ cell elimination was poorly tolerated, the mice had to be euthanized after a short treatment window, with only modest effects on tumor growth observed (Figures 7A and Supplemental Figure 7, B and C).

Analysis of draining lymph nodes confirmed that A2V+aCD40 activated DCs, as determined by increased expression of the costimulatory receptor CD86, and promoted the accumulation of $\mathrm{CD}^{+} \mathrm{T}$ cells in both tumor models (Figure 7B). We also observed an expansion of splenic $\mathrm{CD} 8{ }^{+} \mathrm{CD} 44^{+} \mathrm{CD} 62 \mathrm{l}^{+}$memory $\mathrm{T}(\mathrm{Tm})$ cells, suggestive of long-lasting effector function. In addition to increasing the number of intraepithelial $\mathrm{CD}^{+} \mathrm{T}$ cells, A2V+aCD40 enhanced the accumulation of $\mathrm{CD}^{+}$IFN- $\gamma^{+} \mathrm{GZMB}^{+} \mathrm{CTLs}$ and increased the expression of the CTL markers perforin (Prfl) and granzyme A (Gzma) in the tumors. As expected, T cell elimination abated expression of the $\mathrm{T}$ cell response markers (Figure $7 \mathrm{C}$ and Supplemental Figure 7, D-F). Most important, T cell elimination fully rescued cancer cell proliferation and abolished cell death in response to $\mathrm{A} 2 \mathrm{~V}+\mathrm{aCD} 40$ (Figure 7, D and $\mathrm{E}$ ).

Loss of $\mathrm{T}$ cells did not alter the changes in vascular pruning or caliber or the mural coverage of APP tumors in response to A2V+aCD40 (Figure 7F and Supplemental Figure 7G), indicating that TILs do not mediate $\mathrm{A} 2 \mathrm{~V}$-induced vascular normalization. Finally, the attrition of tumor stroma induced by A2V+aCD40, as analyzed by staining for $\alpha$-SMA and expression of the TGF- $\beta$ targets Pail and Ctgf, was also largely T cell independent (Figure $7 \mathrm{G}$ and Supplemental Figure $7 \mathrm{H})$. Overall, our results indicate that the antitumoral activity of A2V+aCD40 is mostly mediated by $\mathrm{T}$ cells in genetic mouse models of MSS CRC, although additional mechanisms must be involved in therapy-induced stromal remodeling and destruction.

Finally, we gauged the requirement of ANGPT2 blockade for $\mathrm{A} 2 \mathrm{~V}+\mathrm{aCD} 40$ therapeutic efficacy. We used B20, a VEGFAblocking antibody equivalent to bevacizumab (58). A2V+aCD40 but not $\mathrm{B} 20+\mathrm{aCD} 40$ promoted infiltration of intratumoral $\mathrm{CD} 8^{+}$ $\mathrm{T}$ cells and induced significant cell death in size-matched AP and APP tumors (Figure 7, H and I). Although no differences were found in blood vessel density (data not shown), only A2V+aCD40 increased blood vessel caliber (indicative of normalization) and depleted the tumor stroma (Supplemental Figure 7I). These results indicate that double blockade of ANGP2 and VEGFA is more efficient in promoting immune activation and cancer cell destruction.

Overall, our results present a model in which vascular normalization by $\mathrm{A} 2 \mathrm{~V}$ prevents the accumulation of immunosuppressive Tregs and facilitates the formation of TLSs, which may function as additional sites of naive $\mathrm{T}$ cell infiltration and $\mathrm{T}$ cell priming (55). Although such effects were not sufficient to induce significant cancer cell killing per se, the combination with a CD40 agonist enabled antigen-presenting cell activation and the destruction of cancer cells by CTLs (Figure 7J).

\section{Discussion}

The molecular mechanisms that drive the emergence of phenotypically distinct CRC subtypes and their differential responses to therapies are currently under intense investigation. Although adjuvant chemotherapy increases overall survival, many patients either do not respond to treatment or relapse. Recent 
A

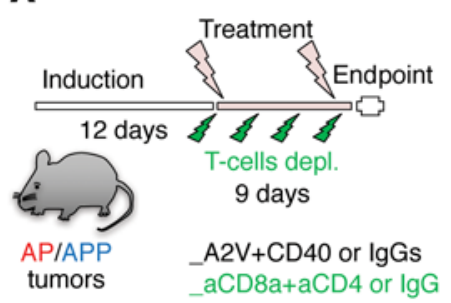

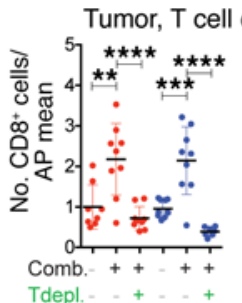

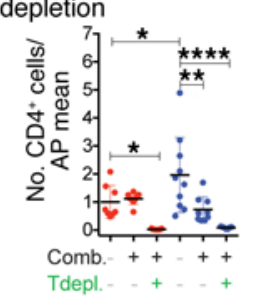

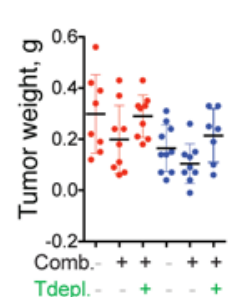

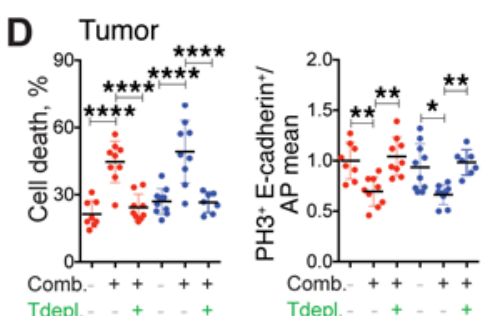

B
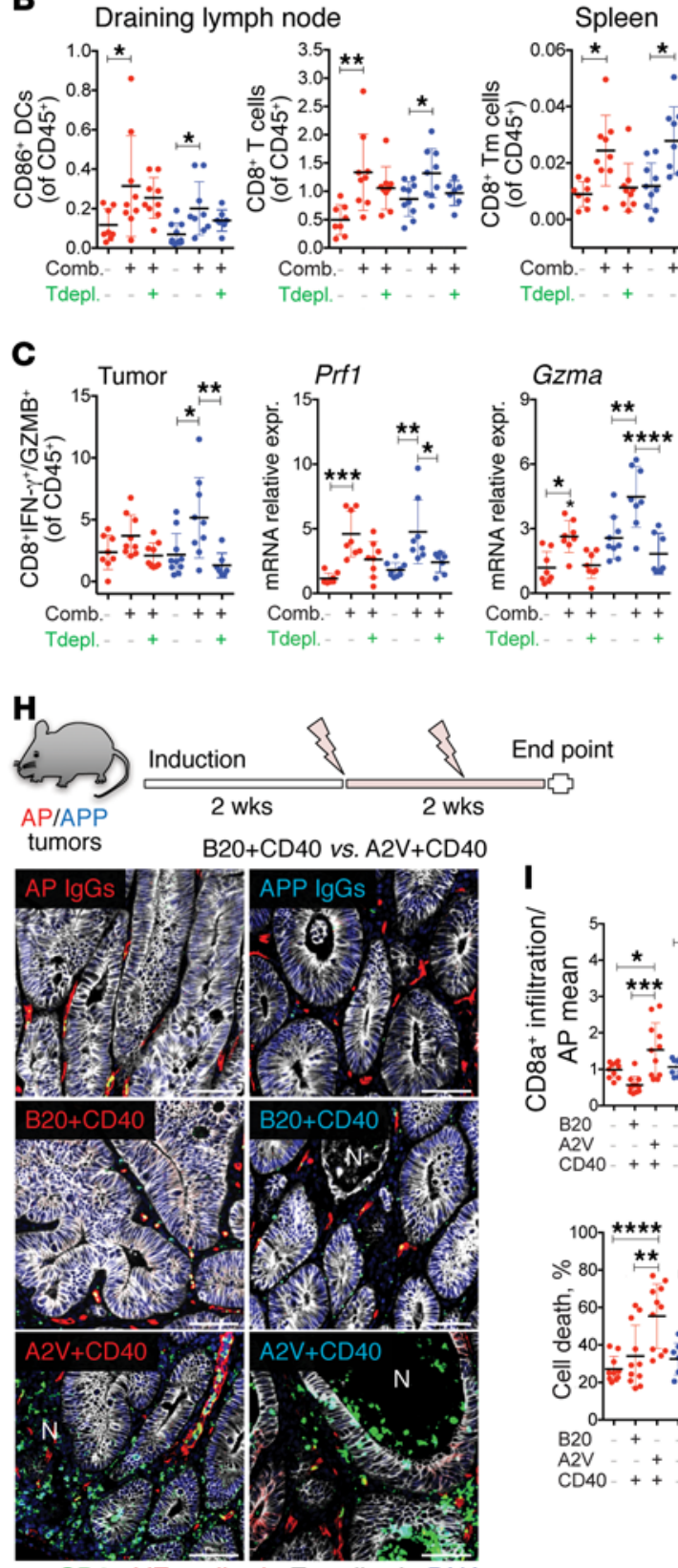

End point

CD8a VE-cadherin E-cadherin DNA
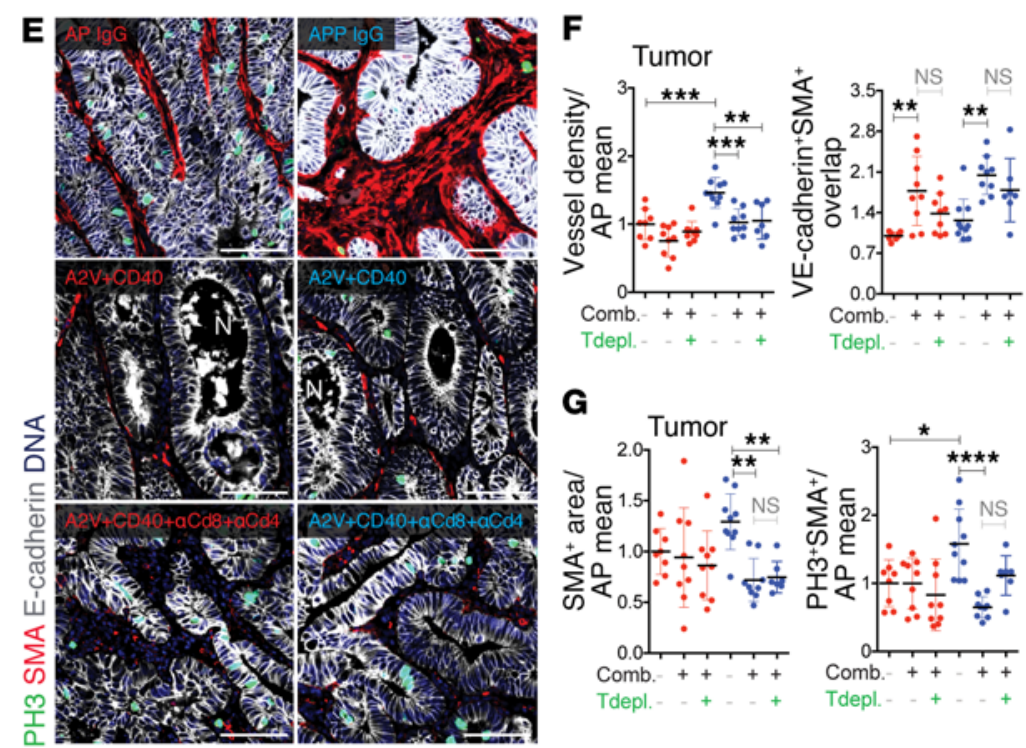

J
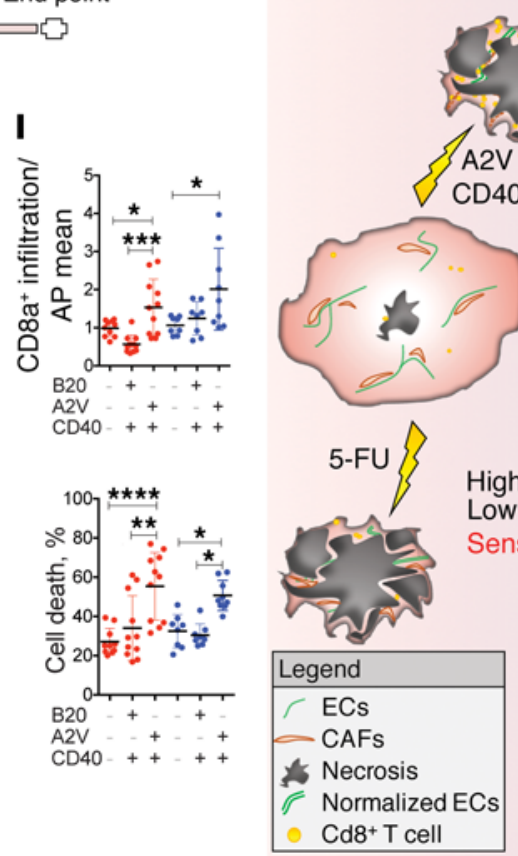

$\checkmark$ CAF activation $\uparrow$.

$\checkmark$ Angiogenesis $\uparrow$ 
Figure 7. A2V+aCD40 antitumor activity is $\mathrm{T}$ cell dependent. (A) Tumors were treated with control mAbs, $\mathrm{A} 2 \mathrm{~V}+\mathrm{aCD} 40$, or $\mathrm{A} 2 \mathrm{~V}+\mathrm{aCD} 40+\alpha-\mathrm{CD} 8+\alpha-$ CD4. Quantification of intratumoral T cells was determined by the fold change versus the AP IgGs mean. AP IgGs, $n=8$; AP A2V+aCD40 AP $\mathrm{A} 2 \mathrm{~V}+\mathrm{aCD} 40+\alpha \mathrm{CD} 8+\alpha \mathrm{CD} 4$ or APP A2V $+a C D 40, n=9$; APP IgGs, $n=10$; APP A2V $+a C D 40+\alpha C D 8+\alpha C D 4, n=7$. (B) FACS analysis of lymph node

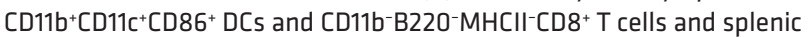
CD11b-B220-MHCII-CD8a+CD44+CD62l ${ }^{+}$Tm cells. (C) Quantification of intratumoral cytotoxicity. Quantification of CD11b-CD3+CD8 $a^{+} I F N-\gamma^{+}$and $\mathrm{CZMB}^{+}$ T cells, percentage of CD45 cells, and qRT-PCR analysis of Prf1 and Gzma expression, normalized to the AP of IgGs mean. (D) T cells mediated cancer cell death in $\mathrm{A} 2 \mathrm{~V}+\mathrm{aCD} 40$-treated tumors. Cell death was determined by the percentage of necrotic glands. $\mathrm{PH}^{+} \mathrm{E}$-cadherin ${ }^{+}$cells were normalized to the AP IgGs mean. (E) Effect of T cell depletion on tumor cell death and stroma. Images show staining for PH3 (green), $\alpha$-SMA (red), E-cadherin (white), and DNA (blue). Scale bars: $50 \mu \mathrm{m}$. (F) Quantification of vessel density and mural coverage. Data were normalized to the AP IgGs mean. (C) A2V+aCD40 reduced stromal content after T cell depletion. Quantification of stromal content and proliferation is shown, and data were normalized to the AP IgGs mean. (H) Comparison of A2V+aCD40 versus $\mathrm{A} 2 \mathrm{~V}+\mathrm{B} 20$ combination treatment. Images show staining for $\mathrm{CD} 8 \mathrm{a}$ (green), VE-cadherin (red), E-cadherin (white), and DNA (blue). AP IgGs, $n=10$; AP $\mathrm{B} 20+\mathrm{aCD} 40$ or AP A2V+aCD40, $n=11$; APP IgGs or APP B20+aCD40, $n=8$; APP A2V $+a C D 40, n=9$. Scale bars: $50 \mu \mathrm{m}$. (I) Quantification of CD8 ${ }^{+} \mathrm{T}$ cell infiltration and cell death. Cell death was determined by the percentage of necrotic tumor glands. CD8 $a^{+} T$ cell infiltration was determined by the fold change versus the AP IgGs mean. (J) PROX ${ }^{\text {hi }}$ tumors display high WNT signaling, a small amount of stroma, and are chemosensitive. Low PROX1 levels blunt WNT activation and promote tumor desmoplasia, angiogenesis, and chemoresistance. $\mathrm{A} 2 \mathrm{~V}+\mathrm{aCD} 40$ normalizes the tumor vasculature, reduces Treg numbers, expands TLSs, and induces antitumor responses. Data represent the mean $\pm \mathrm{SD}$. ${ }^{*} P \leq 0.05$, ${ }^{*} P \leq 0.01$, ${ }^{* * *} P \leq 0.001$, and ${ }^{* * * *} P \leq 0.0001$, by 1 -way ANOVA with Tukey's multiple comparisons test. Comb., combination; Tdepl., T cell depletion.

clinical evidence indicates that epithelium-rich tumors with high WNT signaling respond best to chemotherapy, whereas outcomes are worst for patients with desmoplastic CRC (7, 10, 11). Increased TGF- $\beta$ signaling or Notch hyperactivation has been shown to promote desmoplasia in CRC models $(14,47,59)$, indicating that multiple pathways contribute to stromal expansion in a subset of MSS CRCs.

Here, we show that high expression of PROX1 correlated with lower stromal content in human CRC. Prox1 inactivation was sufficient to promote tumor fibrosis, angiogenesis, and chemoresistance in the genetic model of CRC. Interestingly although the same APC mutations were present in all cancer cells, areas with both high and low intratumoral WNT signaling were observed in mouse and human $A P C$-mutant tumors (this study and ref. 60), indicating that additional mechanisms control WNT activation in CRC. PROX1 is induced in CRC cells only in response to a high and sustained WNT activation (22). Conversely, we report here that loss of Prox 1 blunted WNT activity and increased the production of multiple profibrotic factors, including TGF- $\beta 1$ and MMP14. Of note, Notch hyperactivation reduces PROX1 expression (61) and generates desmoplastic, invasive CMS4-like tumors with high TGF- $\beta$ signaling (59), whereas PROX1 suppresses Notch signaling in colon cancer cells (62). Taken together, these observations suggest a model in which fluctuations of intratumoral Notch and WNT activity, if sufficient to switch off PROX1, will initiate a conversion toward a distinct tumor phenotype, in which cancer cells still slowly proliferate but also promote TGF- $\beta$-dependent stromal activation and angiogenesis (Figure 7J).

High stromal content also fosters the chemoresistant phenotype of Prox1-defcient tumors, because chemotherapy fuels stromal cell activation and angiogenesis, which then support the survival of tumor cells. Interestingly, 5-FU enhanced the growth of orthotopic APP tumors, whereas the growth of s.c. tumors was not affected. Of note, the site of tumor implantation may influence tumor growth and its response to therapy (63). Furthermore, dermal and intestinal fibroblasts display distinct responses to cytokines (64), thus they may differentially contribute to the tumor response to chemotherapy.

Mechanistically, MMP14, which is repressed by PROX1 (ref. 48 and this study), recapitulated several features of Prox1-deficient tumors, including desmoplasia, angiogenesis, and chemoresistance. MMP14 promotes invasion in several cancers through its ability to degrade collagen and other ECM components (65), thus the increased fibrosis in MMP14-AKP tumors may appear counterintuitive. However, MMP14 also promotes fibrosis by cleaving latent TGF- $\beta$. Thus, the function of MMP14 in the TME is more complex and may include not only ECM degradation but also enhanced release of profibrotic factors and ECM production and assembly. Of note, inhibition of MMP14 reduced TGF- $\beta$ production and increased tumor perfusion in breast carcinoma models (66), and we observed a strong potentiation of MMP14 expression in both cancer and stromal cells in response to chemotherapy, along with enhanced fibrosis. Antibody-mediated MMP14 blockade has shown promising results in limiting the progression of mammary tumor models (66). In the future, it will be interesting to explore the clinical relevance of our findings by studying whether MMP14 inhibition sensitizes APP tumors to 5 -FU or further potentiates the antitumoral activity resulting from aCD $40+\mathrm{A} 2 \mathrm{~V}$ treatment.

In our search for an alternative treatment for chemoresistant CRC, we investigated the potential of immune therapy. Blockade of PD-1/PD-L1 signaling targets exhausted $\mathrm{CD} 8^{+} \mathrm{T}$ cells. It is effective in highly mutated MSI CRCs, in which the $\mathrm{T}$ cell response has already been elicited $(67,68)$. To date, all immune interventions, including PD-1/PD-L1 checkpoint blockade, adoptive T cell transfer, and vaccination, have failed in human MSS CRCs. The specific cellular and molecular mechanisms are only beginning to be understood. Notably, in mouse MSS intestinal tumors, which are unresponsive to $\alpha-\mathrm{PD}-1$ therapy, blockade of TGF- $\beta$ was able to initiate a productive $\mathrm{T}$ cell response (14). Here, we investigated an alternative combinatorial approach that targeted tumor blood vessels and CD40 signaling. CD4OL/CD40 is a costimulatory pathway that promotes antigen-presenting cell activation and $\mathrm{CD} 8^{+} \mathrm{T}$ cell-mediated antitumor immunity $(21,57)$. While PD-1 blockade induces tumor rejection by restoring the activity of exhausted $\mathrm{CD} 8^{+}$effector $\mathrm{T}$ cells, stimulation of $\mathrm{CD} 40$ activates DCs and enhances $\mathrm{T}$ cell priming. Moreover, $\mathrm{CD} 4 \mathrm{O}$ activation also directly boosts tumor-specific $\mathrm{CD} 8^{+} \mathrm{T}$ cell immunity and thus may be complementary to immune checkpoint blockade approaches $(57,69)$. Our results show that the antitumor efficacy of aCD40 monotherapy is limited, as observed in other tumor models and in clinical trials $(20,56,70)$. In contrast, additional blockade of VEGFA and ANGPT2, a vessel-destabilizing ligand of TIE2 and a 
regulator of blood vessel maturation, potently increased the ability of aCD40 to stimulate an antitumor immune response. In preclinical models of breast cancer, the blockade of ANGPT2 potentiates the effects of VEGFA inhibition on tumor growth, inhibits metastasis, and sensitizes tumors to PD-1 checkpoint blockade (17). Our results indicate that double blockade of ANGPT2 and VEGFA is also more effective than $\alpha$-VEGFA in promoting immune activation and cancer cell destruction in CRC models in response to CD40 activation.

Systemic aCD40 therapy is associated with dose-limiting toxicities (refs. 71, 72 and this study, Supplemental Figure 7B), therefore, we were unable to evaluate whether repeated administration of $\mathrm{A} 2 \mathrm{~V}+\mathrm{aCD} 40$ could suppress tumor growth for more extended time periods. Intratumoral- or ECM-targeted delivery of aCD40 antibodies proved highly effective and devoid of systemic toxicity in s.c. sarcoma and melanoma models $(72,73)$. These studies provide a rationale for testing tumor-targeted aCD40 antibodies and evaluating the long-term benefits of the combined treatment in our CRC models. Also, an important question relates to the cell type(s) targeted by the aCD40 antibody in AP and APP models. Previous studies documented the important role of CD40 in tumorassociated macrophages, DCs (71), and B cells (74). Interestingly, we found that in both AP and APP tumors, CD40 was prominently expressed in a subset of normal and cancer-associated fibroblasts, suggesting that these cells may potentially contribute to the antitumor activity of aCD40 (Supplemental Figure 8).

Antiangiogenic blockade affects tumor immunity on several levels. Vascular normalization improves the alignment of ECs and pericytes and vessel functionality and promotes CTL infiltration and activity by increasing the expression of endothelial adhesion receptors for T cell trafficking, thereby reducing hypoxia and relieving high interstitial fluid pressure (75). In addition, enhanced tumor cell death caused by an insufficient supply of oxygen and nutrients upon angiogenesis inhibition and the subsequent release of tumorassociated antigens may stimulate antitumor immune responses. We observed no effect (AP model) or a modest decrease (APP mod$\mathrm{el}$ ) in the vascular density of $\mathrm{A} 2 \mathrm{~V}$-treated tumors, and $\mathrm{A} 2 \mathrm{~V}$ alone had no significant effect on tumor cell proliferation or tumor necrosis. Furthermore, both AP and APP tumors displayed low hypoxia, which was not increased after antiangiogenic therapy. These observations argue that dual VEGFA and ANGPT2 blockade in these CRC models does not alter vascular perfusion or intratumoral hypoxia, but rather enhances the maturation of the tumor vascular network in a manner that promotes better lymphocyte trafficking through, for example, increased expression of lymphocyte chemokines or adhesion receptors by endothelial or mural cells.

Indeed, the most consistent effects of $\mathrm{A} 2 \mathrm{~V}$ in both models were an enhanced recruitment of pericytes, the formation of TLSs, reduced stromal activation, an accumulation of Tregs, and an increased infiltration of CTLs. The role of Tregs in human CRC is controversial: high intratumoral Treg density has been associated with better outcomes $(76,77)$, whereas other data indicate that Tregs promote protumor Th17 responses and suppress tumorspecific $\mathrm{CD}^{+} \mathrm{T}$ cell activation in patients with CRC (78). It is possible that Tregs play a beneficial, anticancer role by reducing local inflammation in the absence of immune therapy, whereas in the presence of immune activation by aCD40, they become harmful by suppressing the antitumor activity of CTLs. Here, we propose that relieving Treg-mediated immunosuppression in combination with the activation of $\mathrm{CD} 8^{+} \mathrm{T}$ cell function by aCD 40 underlies the enhancement of $\mathrm{T}$ cell immunity by A2V+aCD 40 .

The destruction of tumor fibrosis, and in particular periepithelial CAFs, by A2V+aCD40 is likely an important additional factor that facilitates engagement of CTLs with cancer cells and increases therapeutic efficacy. The attrition of tumor stroma by $\mathrm{A} 2 \mathrm{~V}+\mathrm{aCD} 40$ was $\mathrm{T}$ cell independent, hence, additional mechanisms such as IFN- $\gamma$-dependent reprogramming of tumor-associated macrophages may be responsible for stromal remodeling by $\mathrm{A} 2 \mathrm{~V}+\mathrm{aCD} 40$, as observed previously in the desmoplastic PDAC model treated with aCD40 (56). Vascular normalization by A2V also promoted the formation of intra- and peritumoral TLSs. The presence of TLSs is a generally favorable prognostic factor in human cancers, and they enhance the local antitumor immune response by providing a site for the homing and activation of naive $\mathrm{T}$ cells (79). TLSs have the advantage of concentrating immune cells and promoting an immune response in close proximity to cancer cells, which might complement or even circumvent the need for immune cell trafficking between the tumor and secondary lymphoid organs. Angiogenic blockade or the direct delivery of the lymphotoxin $\beta$ receptor ligand LIGHT to tumor vessels in a genetic model of pancreatic neuroendocrine tumor promoted the formation of HEV-like vessels or de novo formation of TLSs and improved the response to immune therapies $(18,19)$. Altogether, our results provide further evidence that vascular normalization by $\mathrm{A} 2 \mathrm{~V}$ preconditions the TME for improved $\mathrm{T}$ cell penetration and function.

In summary, our results uncover a mechanism underlying chemoresistance in CRC by showing that low PROX1 expression in Apc-mutant cancer cells generates desmoplastic and chemoresistant tumors. We further propose that chemotherapy is potentially harmful in patients with desmoplastic tumors, as in mouse models, it led to TGF- $\beta$ - and MMP14-dependent stromal activation and angiogenesis and fostered a cancer-supporting stromal microenvironment. We also show that simultaneous vascular normalization by A2V and immune activation by aCD40 had substantial therapeutic activity and efficiently inhibited both chemosensitive and chemoresistant $A p c$-mutant intestinal tumors. We believe these results call for further investigation of this therapeutic approach in human MSS CRCs.

\section{Methods}

A detailed description of the methods is available in the Supplemental Methods.

Statistics. Statistical analysis was performed using GraphPad Prism 6 for MacOS (GraphPad Software). A Student's $t$ test or ordinary 1-way or 2-way ANOVA with Tukey's multiple comparisons test was performed, with the 1-way ANOVA used for grouped comparisons and 2-way ANOVA for pairwise comparisons. Linear regression with a 95\% CI was used to evaluate correlations between 2 variables. Unless otherwise specified, all ex vivo and in vitro data are represented by scatter dot plots with the mean $\pm \mathrm{SD}$, where each individual mouse or cell population repeat is represented by a single dot. In vivo tumor volume growth curves represent the mean value of all individuals from a group $\pm S D$, at each time point. All in vitro analyses were repeated at 
least in triplicate. Statistical significance is indicated as ${ }^{*} P \leq 0.05,{ }^{* *} P$ $\leq 0.01,{ }^{* * *} P \leq 0.001$, and ${ }^{* * *} P \leq 0.0001$, unless otherwise shown in the figure.

RNA-Seq data. RNA-Seq data are deposited in the NCBI's Gene Expression Omnibus (GEO) database (GEO GSE124716).

Study approval. In vivo experiments in animals were approved by the Animal Ethics Committee of Vaud, Switzerland. Human tissue samples were collected at the Triemli Hospital in Zurich, Switzerland, and the Poliambulanza Hospital in Brescia, Italy, with IRB approval of these institutions.

\section{Author contributions}

TVP and SR conceived the study. SR, BPL, AGL, SC, NG, GM, MLS, AG, LW, and JK performed experiments. SR, AGL, BPL, MLS, SN, DB, SC, MPL, and JP analyzed the data. SR and AGL prepared the figures. $\mathrm{SN}, \mathrm{DB}$, and $\mathrm{MD}$ performed bioinformatics analysis. MDP, TVP, EC, and RB designed and supervised the $\mathrm{A} 2 \mathrm{~V}+\mathrm{CD} 40$ studies. TVP, MDP, and SR wrote the manuscript. TVP coordinated the study. All authors reviewed the manuscript.

\section{Acknowledgments}

We thank S. Robine for providing villin-Cre $e^{E R T 2}$ mice; C. Beauverd for mouse genotyping, colony maintenance, and immunostaining; and A. Sabine for mouse strain follow-up. We gratefully acknowledge the assistance of members of the Genomics Technologies, Mouse Pathology, Flow Cytometry, Animal, and Cellular Imaging Facilities of the University of Lausanne. This work was supported by the Medic Foundation (to TVP); the Swiss League for Cancer Research (KFS-3933-08-2016 and KFS-4895-08-2019, to TVP, and KFS-3759-08-2015, to MDP); Roche (to TVP and MDP); the Fondation de Lutte Contre le Cancer (to TVP); the San Salvatore Foundation (to TVP;) the Swiss National Science Foundation No. 310030_179477 (to GM); and the Swiss Bridge Foundation (to TVP and MDP).

Address correspondence to: Tatiana V. Petrova, Department of Oncology, CHUV-UNIL, Ch. des Boveresses 155, CH-1066 Epalinges, Switzerland. Phone: 41.21.314.2968; Email: tatiana. petrova@unil.ch.

NG's present address is: Roche Innovation Center Basel, Roche Pharmaceutical Research and Early Development, pRED, Basel, Switzerland.

EC's present address is Boehringer-Ingelheim Pharmaceuticals, Inc., Ridgefield, Connecticut, USA.
1. Brenner H, Kloor M, Pox CP. Colorectal cancer. Lancet. 2014;383(9927):1490-1502.

2. Van Cutsem E, et al. ESMO consensus guidelines for the management of patients with metastatic colorectal cancer. Ann Oncol. 2016;27(8):1386-1422.

3. Kather JN, Halama N, Jaeger D. Genomics and emerging biomarkers for immunotherapy of colorectal cancer. Semin Cancer Biol. 2018;52(Pt 2):189-197.

4. Guinney J, et al. The consensus molecular subtypes of colorectal cancer. Nat Med. 2015;21(11):1350-1356.

5. Dunne PD, et al. Cancer-cell intrinsic gene expression signatures overcome intratumoural heterogeneity bias in colorectal cancer patient classification. Nat Commun. 2017;8:15657.

6. Isella C, et al. Selective analysis of cancer-cell intrinsic transcriptional traits defines novel clinically relevant subtypes of colorectal cancer. Nat Commun. 2017;8:15107.

7. Allen WL, et al. Transcriptional subtyping and CD8 immunohistochemistry identifies poor prognosis stage II/III colorectal cancer patients who benefit from adjuvant chemotherapy [published online June 13, 2018]. JCO Precis Oncol. https://doi.org/10.1200/PO.17.00241.

8. Calon A, et al. Stromal gene expression defines poor-prognosis subtypes in colorectal cancer. Nat Genet. 2015;47(4):320-329.

9. Isella C, et al. Stromal contribution to the colorectal cancer transcriptome. Nat Genet. 2015;47(4):312-319.

10. Hutchins GGA, et al. Intratumoral stromal morphometry predicts disease recurrence but not response to 5-fluorouracil-results from the QUASAR trial of colorectal cancer. Histopathology. 2018;72(3):391-404.

11. Roepman $\mathrm{P}$, et al. Colorectal cancer intrinsic subtypes predict chemotherapy benefit, deficient mismatch repair and epithelial-to-mesenchymal transition. Int J Cancer. 2014;134(3):552-562.

12. Mlecnik B, et al. Integrative Analyses of Colorectal Cancer Show Immunoscore Is a Stronger Predictor of Patient Survival Than Microsatellite Instability. Immunity. 2016;44(3):698-711.

13. Schumacher TN, S chreiber RD. Neoantigens in cancer immunotherapy. Science. 2015;348(6230):69-74.

14. Tauriello DVF, et al. TGF $\beta$ drives immune evasion in genetically reconstituted colon cancer metastasis. Nature. 2018;554(7693):538-543.

15. De Palma M, Biziato D, Petrova TV. Microenvironmental regulation of tumour angiogenesis. Nat Rev Cancer. 2017;17(8):457-474.

16. Huang Y, Kim BYS, Chan CK, Hahn SM, Weiss man IL, Jiang W. Improving immune-vascular crosstalk for cancer immunotherapy. Nat Rev Immunol. 2018;18(3):195-203.

17. Schmittnaegel M, et al. Dual angiopoietin-2 and VEGFA inhibition elicits antitumor immunity that is enhanced by PD-1 checkpoint blockade. Sci Transl Med. 2017;9(385):eaak9670.

18. Johansson-Percival A, et al. De novo induction of intratumoral lymphoid structures and vessel normalization enhances immunotherapy in resistant tumors. Nat Immunol. 2017;18(11):1207-1217.

19. Allen $\mathrm{E}$, et al. Combined antiangiogenic and antiPD-L1 therapy stimulates tumor immunity through HEV formation. Sci Transl Med. 2017;9(385):eaak9679.

20. Beatty GL, et al. CD40 agonists alter tumor stroma and show efficacy against pancreatic carcinoma in mice and humans. Science. 2011;331(6024):1612-1616.

21. Byrne KT, Vonderheide RH. CD40 Stimulation Obviates Innate Sensors and Drives T Cell Immunity in Cancer. Cell Rep. 2016;15(12):2719-2732.
22. Petrova TV, et al. Transcription factor PROX1 induces colon cancer progression by promoting the transition from benign to highly dysplastic phenotype. Cancer Cell. 2008;13(5):407-419.

23. Lavado A, Lagutin OV, Chow LM, Baker SJ, Oliver G. Prox1 is required for granule cell maturation and intermediate progenitor maintenance during brain neurogenesis. PLoS Biol. 2010;8(8):e1000460.

24. Risebro CA, et al. Prox1 maintains muscle structure and growth in the developing heart. Development. 2009;136(3):495-505.

25. Johnson NC, et al. Lymphatic endothelial cell identity is reversible and its maintenance requires Prox1 activity. Genes Dev. 2008;22(23):3282-3291.

26. Petrova TV, et al. Lymphatic endothelial reprogramming of vascular endothelial cells by the Prox-1 homeobox transcription factor. $E M B O J$. 2002;21(17):4593-4599.

27. Seth A, et al. Prox1 ablation in hepatic progenitors causes defective hepatocyte specification and increases biliary cell commitment. Development. 2014;141(3):538-547.

28. Ragusa S, et al. PROX1 promotes metabolic adaptation and fuels outgrowth of Wnt(high) metastatic colon cancer cells. Cell Rep. 2014;8(6):1957-1973.

29. Wiener Z, et al. Prox1 promotes expansion of the colorectal cancer stem cell population to fuel tumor growth and ischemia resistance. Cell Rep. 2014;8(6):1943-1956.

30. Marisa L, et al. Gene expression classification of colon cancer into molecular subtypes: characterization, validation, and prognostic value. PLOS Med. 2013;10(5):e1001453.

31. Skog M, et al. Expression and prognostic value of transcription factor PROX1 in colorectal cancer. Br JCancer. 2011;105(9):1346-1351. 
32. van de Wetering M, et al. The beta-catenin/ TCF- 4 complex imposes a crypt progenitor phenotype on colorectal cancer cells. Cell. 2002;111(2):241-250.

33. Sansom OJ, et al. Loss of Apc in vivo immediately perturbs Wnt signaling, differentiation, and migration. Genes Dev. 2004;18(12):1385-1390.

34. Barker $\mathrm{N}$, et al. Identification of stem cells in small intestine and colon by marker gene Lgr5. Nature. 2007;449(7165):1003-1007.

35. Batlle E, et al. Beta-catenin and TCF mediate cell positioning in the intestinal epithelium by controlling the expression of EphB/ephrinB. Cell. 2002;111(2):251-263.

36. Hovanes K, et al. Beta-catenin-sensitive isoforms of lymphoid enhancer factor-1 are selectively expressed in colon cancer. Nat Genet. 2001;28(1):53-57.

37. Wielenga VJ, et al. Expression of CD44 in Apc and Tcf mutant mice implies regulation by the WNT pathway. Am J Pathol. 1999;154(2):515-523.

38. Todaro M, et al. CD44v6 is a marker of constitutive and reprogrammed cancer stem cells driving colon cancer metastasis. Cell Stem Cell. 2014;14(3):342-356.

39. Öhlund D, Elyada E, Tuveson D. Fibroblast heterogeneity in the cancer wound. J Exp Med. 2014;211(8):1503-1523.

40. De Roock W, De Vriendt V, Normanno N, Ciardiello F, Tejpar S. KRAS, BRAF, PIK3CA, and PTEN mutations: implications for targeted therapies in metastatic colorectal cancer. Lancet Oncol. 2011;12(6):594-603.

41. Muzumdar MD, Tasic B, Miyamichi K, Li L, Luo L. A global double-fluorescent Cre reporter mouse. Genesis. 2007;45(9):593-605.

42. Lu P, Weaver VM, Werb Z. The extracellular matrix: a dynamic niche in cancer progression. J Cell Biol. 2012;196(4):395-406.

43. Gjorevski N, et al. Designer matrices for intestinal stem cell and organoid culture. Nature. 2016;539(7630):560-564.

44. Romano G, et al. The TGF- $\beta$ pathway is activated by 5 -fluorouracil treatment in drug resistant colorectal carcinoma cells. Oncotarget. 2016;7(16):22077-22091.

45. Tanaka-Nozaki M, Onda M, Tanaka N, Kato S. Variations in 5-fluorouracil concentrations of colorectal tissues as compared with dihydropyrimidine dehydrogenase (DPD) enzyme activities and DPD messenger RNA levels. Clin Cancer Res. 2001;7(9):2783-2787.

46. Grivennikov S, et al. IL-6 and Stat 3 are required for survival of intestinal epithelial cells and development of colitis-associated cancer. Cancer Cell. 2009;15(2):103-113.

47. Calon A, et al. Dependency of colorectal cancer on a TGF- $\beta$-driven program in stromal cells for metastasis initiation. Cancer Cell. 2012;22(5):571-584.

48. Gramolelli S, et al. PROX1 is a transcriptional regulator of MMP14. Sci Rep. 2018;8(1):9531.

49. Kienast Y, et al. Ang-2-VEGF-A CrossMab, a novel bispecific human IgG1 antibody blocking
VEGF-A and Ang-2 functions simultaneously, mediates potent antitumor, antiangiogenic, and antimetastatic efficacy. Clin Cancer Res. 2013;19(24):6730-6740.

50. Rolink A, Melchers F, Andersson J. The SCID but not the RAG-2 gene product is required for S mu-S epsilon heavy chain class switching. Immunity. 1996;5(4):319-330.

51. Lazarus J, et al. Spatial and phenotypic immune profiling of metastatic colon cancer. JCI Insight. 2018;3(22):121932.

52. Nishikawa H, Sakaguchi S. Regulatory T cells in tumor immunity. Int J Cancer. 2010;127(4):759-767.

53. Subramanian A, et al. Gene set enrichment analysis: a knowledge-based approach for interpreting genome-wide expression profiles. Proc Natl Acad Sci U S A. 2005;102(43):15545-15550.

54. de Graaf CA, et al. Haemopedia: An Expression Atlas of Murine Hematopoietic Cells. Stem Cell Reports. 2016;7(3):571-582.

55. Engelhard VH, Rodriguez AB, Mauldin IS, Woods AN, Peske JD, Slingluff CL. Immune cell infiltration and tertiary lymphoid structures as determinants of antitumor immunity. JImmunol. 2018;200(2):432-442.

56. Long KB, Gladney WL, Tooker GM, Graham K, Fraietta JA, Beatty GL. IFN $\gamma$ and CCL2 cooperate to redirect tumor-infiltrating monocytes to degrade fibrosis and enhance chemotherapy efficacy in pancreatic Carcinoma. Cancer Discov. 2016;6(4):400-413.

57. Vonderheide RH, Glennie MJ. Agonistic CD40 antibodies and cancer therapy. Clin Cancer Res. 2013;19(5):1035-1043.

58. Liang WC, et al. Cross-species vascular endothelial growth factor (VEGF)-blocking antibodies completely inhibit the growth of human tumor xenografts and measure the contribution of stromal VEGF. J Biol Chem. 2006;281(2):951-961.

59. Jackstadt R, et al. Epithelial NOTCH signaling rewires the tumor microenvironment of colorectal cancer to drive poor-prognosis subtypes and metastasis. Cancer Cell. 2019;36(3):319-336.e7.

60. Brabletz T, et al. Variable beta-catenin expression in colorectal cancers indicates tumor progression driven by the tumor environment. Proc Natl Acad Sci U S A . 2001;98(18):10356-10361.

61. Kim HA, et al. Notch1 counteracts WNT/ $\beta$-catenin signaling through chromatin modification in colorectal cancer. J Clin Invest. 2012;122(9):3248-3259.

62. Högström J, et al. Transcription factor PROX1 suppresses Notch pathway activation via the nucleosome remodeling and deacetylase complex in colorectal cancer stem-like cells. Cancer Res. 2018;78(20):5820-5832.

63. Blouw B, et al. The hypoxic response of tumors is dependent on their microenvironment. Cancer Cell. 2003;4(2):133-146.

64. West NR. Coordination of immune-stroma crosstalk by IL- 6 family cytokines. Front Immunol. 2019;10:1093.

65. Turunen SP, Tatti-Bugaeva O, Lehti K. Membrane-type matrix metalloproteases as diverse effectors of cancer progression. Biochim Biophys Acta Mol Cell Res. 2017;1864(11 Pt A):1974-1988.

66. Ager EI, et al. Blockade of MMP14 activity in murine breast carcinomas: implications for macrophages, vessels, and radiotherapy. J Natl Cancer Inst. 2015;107(4):djv017.

67. Le DT, et al. Mismatch repair deficiency predicts response of solid tumors to $\mathrm{PD}-1$ blockade. Science. 2017;357(6349):409-413.

68. Le DT, et al. A blueprint to advance colorectal cancer immunotherapies. Cancer Immunol Res. 2017;5(11):942-949.

69. Vonderheide RH. The immune revolution: a case for priming, not checkpoint. Cancer Cell. 2018;33(4):563-569.

70. Furman RR, Forero-Torres A, Shustov A, Drachman JG. A phase I study of dacetuzumab (SGN-40, a humanized anti-CD40 monoclonal antibody) in patients with chronic lymphocytic leukemia. Leuk Lymphoma. 2010;51(2):228-235.

71. Vonderheide RH. CD40 agonist antibodies in cancer immunotherapy [published online August 14, 2019]. Annu Rev Med. https://doi. org/10.1146/annurev-med-062518-045435.

72. Ishihara J, et al. Improving efficacy and safety of agonistic anti-CD40 antibody through extracellular matrix affinity. Mol Cancer Ther. 2018;17(11):2399-2411.

73. Fransen MF, Sluijter M, Morreau H, Arens R, Melief CJ. Local activation of CD8 T cells and systemic tumor eradication without toxicity via slow release and local delivery of agonistic CD40 antibody. Clin Cancer Res. 2011;17(8):2270-2280.

74. Hanissian SH, Geha RS. Jak3 is associated with CD40 and is critical for CD40 induction of gene expression in B cells. Immunity. 1997;6(4):379-387.

75. Lanitis E, Irving M, Coukos G. Targeting the tumor vasculature to enhance T cell activity. Curr Opin Immunol. 2015;33:55-63.

76. Salama $\mathrm{P}$, et al. Tumor-infiltrating $\mathrm{FOXP}^{+} \mathrm{T}$ regulatory cells show strong prognostic significance in colorectal cancer. J Clin Oncol. 2009;27(2):186-192.

77. Frey DM, et al. High frequency of tumor-infiltrating FOXP3(+) regulatory T cells predicts improved survival in mismatch repair-proficient colorectal cancer patients. Int J Cancer. 2010;126(11):2635-2643.

78. Timperi E, et al. Regulatory T cells with multiple suppressive and potentially pro-tumor activities accumulate in human colorectal cancer. Oncoimmunology. 2016;5(7):e1175800.

79. Peske JD, Thompson ED, Gemta L, Baylis RA, Fu YX, Engelhard VH. Effector lymphocyte-induced lymph node-like vasculature enables naive T-cell entry into tumours and enhanced antitumour immunity. Nat Commun. 2015;6:7114.

80. Yoshihara K, et al. Inferring tumour purity and stromal and immune cell admixture from expression data. Nat Commun. 2013;4:2612.

81. Barbie DA, et al. Systematic RNA interference reveals that oncogenic KRAS-driven cancers require TBK1. Nature. 2009;462(7269):108-112. 